\title{
Derivation of a Unique Body Surface Area (Bsa) Formula for Calculation of Relatively Safe Doses of Dog and Human Anticancer Drugs
}

Saganuwan Alhaji Saganuwan*

Department of Veterinary Physiology, Pharmacology and Biochemistry, College of Veterinary Medicine, University of Agriculture, Makurdi, Benue State, Nigeria

\begin{abstract}
The safety of anticancer dosing has become a serious concern due to high incidence of life-threatening toxicity signs. More so, dogs are used as models of research for human cancers. In view of these a uniform body surface area (BSA) formula has been derived for human and dog with a view to having low, safe, effective therapeutic doses of anticancers. The derived formula $\left(B S A=\mathrm{BW}^{0.528} \times \mathrm{H}^{0.528} \times \mathrm{K}\right.$ ) was used to calculate BSAs of greyhound, toy, companion, terrier, hunting and working dogs and yielded low effective therapeutic doses of dacarbazine, asparaginase, streptozotocin, dactinomycin, epirubicine and prednisolone. Hunting and working dogs have high body weight, BMI and BSAs similar to that of human and may be prone to obesity and obesity associated diseases. Whereas BSAs and doses of anticancer agents of light and relatively tall dogs are relatively higher in comparison with that of short and light dogs. Greyhounds have higher BSA in comparison with toys, companions and terriers. Working breeds of dog such as Treeing Walker Coon haired $(65.0 \mathrm{~kg})$, Great Swiss mountain dog $(59.0 \mathrm{~kg})$, longhaired St. Bernard $(55.0 \mathrm{~kg})$, French Mastiff $(50.0 \mathrm{~kg})$ and female Komondor $(59.0 \mathrm{~kg})$ have same BSA values with humans weighing $51.3,46.7,44.8,44.0$ and $43.0 \mathrm{~kg}$, respectively. Calculated common exponent (0.528) for body weight and height may be the common relationship between basal metabolism of dog and human.
\end{abstract}

Keywords: Complex analysis; Body surface area formula; Boyd; Human; Dog; Cancer relapse; Toxicity

Abbreviations: BSA: Body Surface Area; BW: Body Weight; K: Metabolism Constant; BMI: Body Mass Index

\section{Introduction}

Ever since the so-called "Peat Bog Dogs" raised by Neolithic Palafitte dwellers, the appearance of new breeds similar to those we know today took place without pause due to natural mutation, climate, environment or crossings engineered by man in the process of domestication. The breeds became ever more numerous and specialized until they reached the point of modern classification, which is based on the aptitude of the breed and the use: hunting, shepherding, guarding, work and company. Perhaps the first fully distinct breed was saluki, an Arabian Greyhound whose name means 'noble' [1]. The concept of breeds was defined by the Arabs first in relating to horses and later to dogs. Aristotle named dogs according to their country of origination. It is estimated that the canine population of the world is $120-150$ million. In the USA alone, there are approximately 35 million dogs, and 7-8 million live in France, 5-6 million in Germany, 4 million in Italy, 1 million in Belgium and 400,000 in Switzerland [1]. Not all of them are happy dogs as result of diseases, abandonment by ungrateful masters and vivisection. Brazil has the largest number of dog population studies [2]. The safety of drug dosing has become a concern, because of high incidence of serious or life-threatening toxicity associated with many anticancer drugs [3]. Allometry is the relationship between the physiological, anatomical and pharmacokinetic parameters and body size. Precise calculation of total body surface area is of great importance in many biomedical applications, most especially for calculation of doses of anticancer agents [4]. In human medicine, the DuBois formula has been recommended in cancer chemotherapy above other formulas [5] followed by Wang et al. formula [6]. Because of a presumed narrow therapeutic index for most anticancer agents as shown in breast cancer, testicular cancer, lymphoma, and other cancers, cancer chemotherapy can be challenging for not only human medical oncologist [7] but also veterinary medical oncologist. However, the formula commonly used for calculation of anticancer doses for dogs is Cowgill and Drabkin's formula [8] which does not put linearity into consideration. Because of high risk of toxicity caused by use of the formula, there is need to compare some human BSA formulas with dog BSA formula with a view to deriving a human-dog equivalent BSA formula. Cancer being exacerbated by the modern life style as revealed by study [9] requires a radical therapeutic approach that put into consideration, the use of BSA that would give less toxic and moderate effective doses of anticancer agents [10]. In view of this, new body surface area formula and doses of twenty-eight anticancer drugs were calculated for 334 breeds of greyhound, toy, companion, terrier, hunting and working dogs.

\section{Materials and Methods}

\section{Derivation of dog BSA from human BSA formulas}

The BSA of randomly selected twelve dogs from ten breeds were initially calculated using the human BSA formulas developed by various authors (Table 1). But for derivation of a new formula for dogs, the height of all the breeds of dogs were multiplied by 2 [11-15]. Ten breeds of dog used in this study are Great Pyrene, New Foundland, Lemberger, Black Russian, Saint Bernard, Neapolitan Mastiff, Great Danes, Scottish Deerhound, Bullmastiff and Wolfhound [16,17]. The new formula was derived from DuBois [5], Haycock et al. [12], Boyd [14] and Fujimoto [16] formulas using average of their body weight and height exponents. Takashira, [15], Wang et al. [6] and Saganuwan and Ndakotsu [10] formulas were not used because they have same values of exponents with DuBois formula. Gehan and George [13] formula was

*Corresponding author: Saganuwan Alhaji Saganuwan, Department of Veterinary Physiology, Pharmacology and Biochemistry, College of Veterinary Medicine, University of Agriculture, P.M.B. 2373, Makurdi, Benue State, Nigeria, Tel: 2348027444269; E-mail: pharn_saga2006@yahoo.com

Received July 11, 2017; Accepted October 27, 2017; Published October 30, 2017

Citation: Saganuwan SA (2017) Derivation of a Unique Body Surface Area (Bsa) Formula for Calculation of Relatively Safe Doses of Dog and Human Anticancer Drugs. J Cancer Sci Ther 9: 690-704. doi:10.4172/1948-5956.1000493

Copyright: ( 2017 Saganuwan SA. This is an open-access article distributed unde the terms of the Creative Commons Attribution License, which permits unrestricted use, distribution, and reproduction in any medium, provided the original author and source are credited. 
Citation: Saganuwan SA (2017) Derivation of a Unique Body Surface Area (Bsa) Formula for Calculation of Relatively Safe Doses of Dog and Human Anticancer Drugs. J Cancer Sci Ther 9: 690-704. doi:10.4172/1948-5956.1000493

not used because it has same body weight and body height exponent with Haycock et al. [12] formula. The derived body weight and height exponent was 0.528 and the constant $(\mathrm{K})$ for dog derived from constant of DuBois, Haycock et al., Boyd and Fujimoto, formulas was $1.4 \times 10^{-1}$. Therefore, the derived new BSA formula for dog is $\mathrm{BSA}=\mathrm{BW}^{0.528} \times \mathrm{H}^{0.528}$ $\times \mathrm{K}$ were $\mathrm{K}$ is $1.4 \times 10^{-1}$.

\section{Application of dog new BSA formula}

BSA formula $\left(\mathrm{BSA}=\left(\mathrm{BW}^{2 / 3}\right) \times 10.1 \times 10^{-4}\right)$ developed by Cowgill and Drabkin [8] and the derived BSA formula $\left(\mathrm{BSA}=\mathrm{BW}^{0.528} \times \mathrm{H}^{0.528}\right.$ $\times \mathrm{K}$ ) were used for calculation of body surface area for greyhounds (12 breeds), toys ( 26 breeds), companions ( 25 breeds), hunting (141 breeds), terriers (28 breeds) and working dogs ( 92 breeds). Doses of doxorubicine, melphalan, vincristine, chlorambucil, cyclophosphamide and carboplatine were calculated for greyhounds. However, bleomycin, busulfan, cisplatin, cytarabine, dacarbazine, dronabinol and lumustine were calculated for toys. Calculated for companions are 6-mercaptopurine, carmustine, fluorouracil, mitoxantrone, thioguanine, streptozotocin and Thio-Tepa. Whereas prednisolone, asparaginase and ifosfamide were calculated for terriers. The doses of methotrexate, dactinomycin, mechlorethamine, Thio-TEPA and mercaptopurine were calculated for hunting dogs. But dacarbazine, asparaginase, streptozotocin, dactinomycin, epirubicine and prednisolone [1822] were calculated for working dogs. Body mass index (BMI) was calculated for all the breeds using human BMI formula $\left(B M I=\frac{\text { Weight }}{\text { Height }^{2}}\right)$ . A total of 334 adult breeds of dogs (7-10 years) comprising 259 males and 75 females were used for the study [18].

\section{Statistical Analysis}

All the data on Table 1 of BSA formulas were presented as mean \pm standard error of mean (SEM). Analysis of Variance (ANOVA) was

\begin{tabular}{|c|c|c|c|c|}
\hline S/No. & Author & Year of Publication & No. of Patients & Formula \\
\hline 1 & Mosteller [11] & 1987 & Modified Gehan and George & $\left(\frac{[H x W]}{3600^{\frac{1}{2}}}\right)$ \\
\hline 2 & DuBois [5] & 1916 & 9 & $2.0247 \times 10^{-1} \times \mathrm{H}^{0.725} \times \mathrm{W}^{0.425}$ \\
\hline 3 & Haycock et al [12] & 1978 & 81 & $2.4265 \times 10^{-2} \times H^{0.4246} \times W^{0.51456}$ \\
\hline 4 & Gehan and George [13] & 1970 & 401 & $2.35 \times 10^{-2} \times \mathrm{H}^{0.4246} \times \mathrm{W}^{0.51456}$ \\
\hline 5 & Boyed [14] & 1935 & 411 & $3.207 \times 10^{-4} \times \mathrm{H}^{0.3} \times \mathrm{W}^{0.7285}$ \\
\hline 6 & Wang et al [6] & 1992 & Modified DuBois & $7.184 \times 10^{-3} \times \mathrm{H}^{0.725} \times \mathrm{W}^{0.425}$ \\
\hline 7 & Takashira [15] & 1925 & Unknown & $7.241 \times 10^{-3} \times \mathrm{H}^{0.725} \times \mathrm{W}^{0.425}$ \\
\hline 8 & Fujimoto [16] & 1968 & 201 & $8.883 \times 10^{-3} \times H^{0.663} \times W^{0.444}$ \\
\hline 9 & Saganuwan and Ndakotsu [10] & 2015 & II-modified DuBois & $2.008 \times 10^{-1} \times \mathrm{H}^{0.725} \times \mathrm{W}^{0.425}$ \\
\hline 10 & Cowgill and Drabkin [8] & 1927 & I-Dog BSA formula & $W \times 10.1 \times 10^{-4}$ \\
\hline 11 & Saganuwan (unpublished) & This paper & Modified DuBois, Haycock et al. Boyd and Fujimoto & $W^{0.528} \times 10^{0.528} \times 1.4 \times 10^{-1}$ \\
\hline
\end{tabular}

Table 1: Body Surface area formulas.

\begin{tabular}{|c|c|c|c|c|c|c|c|c|c|c|c|c|c|c|}
\hline $\begin{array}{l}\text { Breed of } \\
\text { dog }\end{array}$ & $\begin{array}{c}\text { Body } \\
\text { weight }(\mathrm{kg})\end{array}$ & $\begin{array}{l}\text { Height } \\
x 2\left(m^{2}\right)\end{array}$ & $\begin{array}{c}\text { Body } \\
\text { index } \\
\left(\mathrm{kg} / \mathrm{m}^{2}\right)\end{array}$ & $\begin{array}{c}\text { Cowgill and } \\
\text { Drabkin } \\
\text { (dog) }\end{array}$ & DuBois & Monsteller & $\begin{array}{c}\text { Haycock } \\
\text { et al. }\end{array}$ & $\begin{array}{l}\text { Gehan } \\
\text { and } \\
\text { George }\end{array}$ & Boyd & $\begin{array}{l}\text { Wang } \\
\text { et al. }\end{array}$ & Takashira & Fujimoto & $\begin{array}{c}\text { Saganuwan } \\
\text { and } \\
\text { Ndakotsu }\end{array}$ & $\begin{array}{l}\text { This } \\
\text { paper }\end{array}$ \\
\hline $\begin{array}{c}\text { Great } \\
\text { Pyrene }\end{array}$ & 54 & 1.52 & 23.37 & 1.49 & 1.49 & 1.37 & $1.58^{a}$ & $1.53^{\mathrm{a}}$ & 1.02 & 1.49 & $1.51^{\mathrm{a}}$ & 1.46 & 1.48 & 1.43 \\
\hline $\begin{array}{c}\text { New } \\
\text { Foundland }\end{array}$ & 68 & 1.42 & 33.72 & 1.74 & 1.53 & 1.61 & 1.72 & 1.67 & 1.18 & 1.57 & 1.58 & 1.55 & 1.56 & 1.56 \\
\hline Lemberger & 54 & 1.42 & 26.78 & 1.49 & 1.42 & 1.28 & $1.53^{\mathrm{a}}$ & $1.53^{\mathrm{a}}$ & 1.00 & 1.42 & 1.43 & 1.40 & 1.41 & 1.38 \\
\hline $\begin{array}{c}\text { Black } \\
\text { Russian }\end{array}$ & 50 & 1.32 & 28.70 & 1.42 & 1.31 & 1.10 & 1.43 & 1.38 & 0.92 & 1.31 & 1.32 & 1.28 & 1.29 & 1.27 \\
\hline $\begin{array}{c}\text { Saint } \\
\text { Bernard }\end{array}$ & 59 & 1.30 & 34.91 & 1.59 & 1.39 & 1.28 & 1.55 & 1.50 & 1.04 & 1.39 & 1.40 & 1.37 & 1.37 & 1.15 \\
\hline $\begin{array}{c}\text { Neapolitan } \\
\text { Mastiff }\end{array}$ & 50 & 1.22 & 33.59 & 1.42 & 1.23 & 1.02 & 1.38 & 1.34 & 0.90 & 1.023 & 1.24 & 1.22 & 1.22 & 1.23 \\
\hline $\begin{array}{l}\text { Great } \\
\text { Danes }\end{array}$ & 59 & 1.72 & 19.94 & 1.59 & 1.69 & 1.69 & 1.74 & 1.69 & 1.13 & 1.70 & 1.71 & 1.65 & 1.57 & 1.61 \\
\hline $\begin{array}{c}\text { Scottish } \\
\text { Deerhound }\end{array}$ & 54 & 1.42 & 26.78 & 1.50 & 1.42 & 1.28 & 1.53 & 1.49 & 1.00 & 1.42 & 1.43 & 1.40 & 1.41 & 1.38 \\
\hline $\begin{array}{l}\text { Bullmastiff } \\
(\mathrm{m})\end{array}$ & 110 & 1.52 & 47.61 & 2.41 & 2.02 & 2.79 & 2.28 & 2.20 & 1.70 & 2.02 & 2.04 & 2.00 & 2.00 & 2.09 \\
\hline $\begin{array}{l}\text { Bullmastiff } \\
\text { (f) }\end{array}$ & 91 & 1.38 & 47.78 & 2.12 & 1.73 & 2.09 & 1.98 & 1.92 & 1.45 & 1.74 & 1.75 & 1.73 & 1.72 & 1.80 \\
\hline $\begin{array}{l}\text { Wolfhound } \\
(\mathrm{m})\end{array}$ & 55 & 1.62 & 20.96 & 1.51 & 1.57 & 1.49 & 1.64 & 1.58 & 1.05 & 1.58 & 1.59 & 1.54 & 1.56 & 1.50 \\
\hline $\begin{array}{l}\text { Wolfhound } \\
\text { (f) }\end{array}$ & 47 & 1.52 & 20.34 & 1.36 & 1.40 & 1.19 & 1.47 & 1.42 & 0.92 & 1.41 & 1.42 & 1.37 & 1.40 & 1.38 \\
\hline$\underset{\text { SEM }}{\text { Mean } \pm}$ & $62.16 \pm 5.5$ & $\begin{array}{l}1.4 \pm \\
0.04\end{array}$ & $30.4 \pm 2.8$ & $1.63 \pm 0.09^{c}$ & $\begin{array}{l}1.51 \pm \\
0.06^{b}\end{array}$ & $1.51 \pm 0.14^{b}$ & $\begin{array}{l}1.65 \pm \\
0.07^{\mathrm{a}}\end{array}$ & $\begin{array}{l}1.60 \pm \\
0.06^{\mathrm{b}}\end{array}$ & $\begin{array}{l}1.10 \pm \\
0.06^{\mathrm{b}}\end{array}$ & $\begin{array}{l}1.52 \pm \\
0.06^{b}\end{array}$ & $\begin{array}{l}1.53 \pm \\
0.06^{b}\end{array}$ & $\begin{array}{l}1.49 \pm \\
0.06^{\mathrm{b}}\end{array}$ & $1.49 \pm 0.06^{b}$ & $\begin{array}{l}1.48 \pm \\
0.08\end{array}$ \\
\hline
\end{tabular}

Table 2: The scoring of body surface area (BSA) formulas $\left(\mathrm{m}^{2}\right)$ for calculation of anticancer agents in dogs. 


\begin{tabular}{|c|c|c|c|c|c|c|c|c|c|c|c|c|c|c|c|c|c|}
\hline \multirow[t]{2}{*}{ Breed } & \multirow{2}{*}{$\begin{array}{c}\text { Body } \\
\text { weight } \\
\text { (kg) }\end{array}$} & \multirow{2}{*}{$\begin{array}{l}\text { Height } \\
x 2\left(m^{2}\right)\end{array}$} & \multirow{2}{*}{$\begin{array}{l}\text { Body } \\
\text { index }\end{array}$} & \multirow{2}{*}{$\begin{array}{l}\text { Cowgill and } \\
\text { Drabkin's } \\
\text { BSA formula }\end{array}$} & \multirow{2}{*}{$\begin{array}{c}\text { New } \\
\text { BSA } \\
\text { formula }\end{array}$} & \multicolumn{2}{|c|}{$\begin{array}{c}\text { Doxorubicin } \\
\left(30 \mathrm{mg} / \mathrm{m}^{2}\right)\end{array}$} & \multicolumn{2}{|c|}{$\begin{array}{l}\text { Melphalan } \\
\left(1.5 \mathrm{mg} / \mathrm{m}^{2}\right)\end{array}$} & \multicolumn{2}{|c|}{$\begin{array}{l}\text { Vincristine } \\
\left(2 \mathrm{mg} / \mathrm{m}^{2}\right)\end{array}$} & \multicolumn{2}{|c|}{$\begin{array}{c}\text { Chlorambucil } \\
\left(3 \mathrm{mg} / \mathrm{m}^{2}\right)\end{array}$} & \multicolumn{2}{|c|}{$\begin{array}{c}\text { Cyclophosphamide } \\
\left(50 \mathrm{mg} / \mathrm{m}^{2}\right)\end{array}$} & \multicolumn{2}{|c|}{$\begin{array}{l}\text { Carboplatin } \\
\left(300 \mathrm{mg} / \mathrm{m}^{2}\right)\end{array}$} \\
\hline & & & & & & CDF & NF & CDF & NF & CDF & $\mathrm{NF}$ & $\mathrm{CDF}$ & NF & CDF & NF & CDF & $\mathrm{NF}$ \\
\hline $\begin{array}{l}\text { Arab } \\
\text { Greyhound } \\
(\mathrm{m})\end{array}$ & 32 & 1.56 & 13.15 & 1.02 & 1.10 & 30.6 & 33 & 1.53 & 1.65 & 2.04 & 2.20 & 3.06 & 3.30 & 51.0 & 55.0 & 303 & 330 \\
\hline $\begin{array}{c}\text { Arab } \\
\text { Greyhound (f) }\end{array}$ & 27 & 1.42 & 13.39 & 0.91 & 0.96 & 27.3 & 28.8 & 1.37 & 1.44 & 1.81 & 1.82 & 2.73 & 2.88 & 45.5 & 48.0 & 273 & 288 \\
\hline $\begin{array}{l}\text { Italian } \\
\text { Greyhound }\end{array}$ & 5 & 0.76 & 8.66 & 0.30 & 0.28 & 9.0 & 8.4 & 0.45 & 0.42 & 0.60 & 0.56 & 0.90 & 0.94 & 15.0 & 14.0 & 90.0 & 84.0 \\
\hline Whippet (m) & 12 & 0.94 & 13.58 & 0.53 & 0.50 & 15.9 & 15.0 & 0.80 & 0.75 & 1.06 & 1.00 & 1.54 & 1.50 & 26.5 & 25.0 & 265 & 150 \\
\hline Whippet (f) & 9 & 88 & 11.62 & 0.44 & 2 & 13.2 & 12.6 & 0.6 & 0.63 & \begin{tabular}{|l|}
0.88 \\
\end{tabular} & 0.84 & 1.32 & 1.26 & 22.0 & 1.0 & 132 & 126 \\
\hline $\begin{array}{l}\text { Deerhound } \\
\quad(\mathrm{m})\end{array}$ & 48 & 1.52 & .76 & 1 & 1.35 & 39.9 & 40.5 & 2.00 & 2.03 & 2.66 & 70 & 3.99 & 4.05 & 66.5 & 67.5 & 399 & 405 \\
\hline Deerhound (f) & 36 & 1.42 & 17.85 & 1.10 & 1.12 & 33.0 & 33.6 & 1.65 & 1.68 & 2.20 & 2.24 & 3.30 & 3.36 & 60.5 & 56.0 & 330 & 336 \\
\hline $\begin{array}{l}\text { Irish Wolf } \\
\text { hound }(m)\end{array}$ & 54 & 1.58 & 21.63 & 1.44 & 1.46 & 43.2 & 43.8 & 2.16 & 2.10 & 2.88 & 2.92 & 4.32 & 4.38 & 72.0 & 73.0 & 432 & 438 \\
\hline $\begin{array}{l}\text { Irish Wolf } \\
\text { hound }(\mathrm{f})^{\star \star}\end{array}$ & 40 & 1.42 & 26.78 & 1.18 & 1.18 & 35.0 & 35.0 & 1.77 & 1.77 & 17.70 & 17.70 & 3.54 & 3.54 & 59.0 & 59.0 & 354 & 354 \\
\hline $\begin{array}{l}\text { Spanish } \\
\text { Greyhound } \\
(\mathrm{m})\end{array}$ & 30 & 1.40 & 15.31 & 0.98 & 1.00 & 29.4 & 30.0 & 1.47 & 1.50 & 1.96 & 2.00 & 2.94 & 3.00 & 44.0 & 55.0 & 294 & 300 \\
\hline $\begin{array}{l}\text { Pharaoh } \\
\text { hound }(m)\end{array}$ & 30 & 1.40 & 15.31 & 0.98 & 1.01 & 29.4 & 30.3 & 1.4 & 1.52 & 1.96 & 2.02 & 2.94 & 3.03 & 49.0 & 50.5 & 294 & 303 \\
\hline $\begin{array}{l}\text { Pharaoh } \\
\text { hound (f) }\end{array}$ & 28 & 1.32 & 16.07 & 0.93 & 0.94 & 27.9 & 28.2 & 1.44 & 1.41 & 1.86 & 1.88 & 2.79 & 2.82 & 46.5 & 47.0 & 279 & 282 \\
\hline Afghan hound & 34 & 1.47 & 15.73 & 1.06 & 1.10 & 31.8 & 33.0 & 1.59 & 1.65 & 2.12 & 2.20 & 3.18 & 3.30 & 53.0 & 55.0 & 318 & 330 \\
\hline Saluki & 30 & 1.42 & 14.88 & 0.98 & 1.01 & 29.4 & 30.3 & 1.47 & 1.52 & 1.96 & 2.02 & 2.94 & 3.03 & 49.0 & 50.5 & 294 & 303 \\
\hline Borzoi (m) & 48 & 1.51 & 21.05 & 1.33 & 1.34 & 39.9 & 40.2 & 2.00 & 2.01 & 2.66 & 2.68 & 3.99 & 4.02 & 66.5 & 67.0 & 399 & 402 \\
\hline $\begin{array}{l}\text { Magyar Agar } \\
(\mathrm{m})\end{array}$ & 31 & 1.41 & 15.59 & 1.00 & 3 & 30.0 & 30.9 & 1.50 & 1.55 & 2.00 & 2.06 & 3.00 & 3.09 & 50 & 51.5 & 300 & 309 \\
\hline $\begin{array}{l}\text { Magyar Agar } \\
\text { (f) }\end{array}$ & 26 & 1.31 & 15.18 & 0.89 & 0.90 & 26.7 & 27.0 & 1.34 & 1.35 & 1.78 & 1.80 & 2.67 & 2.70 & 44.5 & 45.0 & 267 & 270 \\
\hline Sloughi & 32 & 1.50 & 14.22 & 1.02 & 1.08 & 30.6 & 32.4 & 1.53 & 1.62 & 2.04 & 2.16 & 3.06 & 3.24 & 51.0 & 54.0 & 306 & 324 \\
\hline
\end{tabular}

CDF: Cowgill and Drabkin's formula; NF: New Formula; m: Male; f: Female; **: Prone to overweight, $\mathrm{BMl}>25 \mathrm{~kg} / \mathrm{m}^{2}$

Table 3: Body weight, height, body mass index, body surface areas and therapeutic doses of anticancer drugs for greyhounds.

used to analyse the data and least significant difference (LSD) was used to detect difference between the calculated BSA values at 5\% level of significance [23].

\section{Results}

Table 2 shows the body surface areas of various breeds of dogs calculated using human BSA formulas. The average weight, height and body mass index of the dogs were $62.16 \pm 5.5 \mathrm{~kg}, 1.4 \pm 0.04 \mathrm{~m}$ and $30.4 \pm 2.8 \mathrm{~kg} / \mathrm{m}^{2}$, respectively. Average BSAs of $1.10 \pm 0.06$ to $1.60 \pm 0.06$ were calculated using the formulas except, Haycock and Cowgill and Drabkin's formulas (Table 2).

Male Irish Wolf hound recorded highest body weight ( $54 \mathrm{~kg})$, height $(0.79 \mathrm{~m})$ and body surface area $\left(1.44-1.46 \mathrm{~m}^{2}\right)$ as Italian Greyhound recorded smallest body weight $(5 \mathrm{~kg})$, height $(0.38 \mathrm{~m})$ and body surface area $\left(0.28-0.30 \mathrm{~m}^{2}\right)$. Fourteen out of 18 breeds of dogs yielded higher BSAs. Wolf hound (female) yielded BSA of same value using the formulas (Table 3 ). The results in Table 4 show that toys generally have low body weight, except female Continental Spaniel and Pekingese breeds that recorded higher body surface areas (BSAs). Body weight, height and body mass index of all the toys are generally low. Companion dogs have low to moderate body weight ranging between $3.0-27.0 \mathrm{~kg}$. Pinscher, Belgian large Griffon and Tibetan Spaniel recorded higher BSAs. Great Spitz, Poodle (miniature) and Chinese Temple dog (miniature) recorded $\mathrm{BMI}>25 \mathrm{~kg} / \mathrm{m}^{2}$, whereas poodle (standard) and French Bulldog recorded BMI $>30 \mathrm{~kg} / \mathrm{m}^{2}$ (Table 5). The terrier dogs recorded low to moderate BSAs and body weights within the range of
$4.5-28.0 \mathrm{~kg}$. However, the formulas yielded same value of BSA and doses of anticancer drugs for Airedale terrier (Table 6). Out of 141 breeds of dogs whose body weight, height and body mass index were calculated, 39 and 23 dogs recorded height $(<1.0 \mathrm{~m})$ and BMI $\left(>30 \mathrm{~kg} / \mathrm{m}^{2}\right)$, respectively. But the formulas yielded same BSA value for Kardian Bear dog, male Billy, Smooth-Haired Istrian Hound, Rodhesian Ridgeback (male), Rhodhesian Ridgeback (female), Bluetick Coon Hound (male), Redtick Coon Hound (male), Drathaar (male), Weimaraner (male), Braque Ariegeois, Braque Daurvergne (female), French Spaniel (female), Briquette and Irish Setter (female). However, Short Haired Dachshound, longhaired Dachshound, Wirehaired Dachshound, Cirneco Dell Etna, female Basenji and Cocker Spaniel yielded weight ranging $8.3-10.9 \mathrm{~kg}$. Majority of hunting dogs recorded BSA of lower values (Table 7). Twenty-two (22) out of 92 working breeds of dogs recorded height less than $1.00 \mathrm{~m}$, whereas 25 dogs recorded $\mathrm{BMI}>30$ $\mathrm{kg} / \mathrm{m}^{2}$. But 5 dogs recorded BSA of same value using the formulas. The dogs are Kuvasz, Maremma Sheepdog, Austrian Shepherd, Doberman Pincher (male and female) and Akita Inu (female). However, Border Collie, Shetland Sheepdog, Iceland dog (female) and Australian Kelpie (female) recorded weight (7-11 kg). Majority of hunting dogs recorded lower BSA values (Table 8).

\section{Discussion}

Allometric scaling popularly applied in prediction of human clearance, based on animal data has errors which could cause either drug toxicity or therapeutic failure. Hence modifications have been 
Citation: Saganuwan SA (2017) Derivation of a Unique Body Surface Area (Bsa) Formula for Calculation of Relatively Safe Doses of Dog and Human Anticancer Drugs. J Cancer Sci Ther 9: 690-704. doi:10.4172/1948-5956.1000493

\begin{tabular}{|c|c|c|c|c|c|c|c|c|c|c|c|c|c|c|c|c|c|c|c|}
\hline \multirow{2}{*}{ Breed } & \multirow{2}{*}{$\begin{array}{c}\text { Body } \\
\text { weight } \\
\text { (kg) }\end{array}$} & \multirow{2}{*}{$\begin{array}{l}\text { Height } \\
\text { x2 (m) }\end{array}$} & \multirow{2}{*}{$\begin{array}{l}\text { Body } \\
\text { index }\end{array}$} & \multirow{2}{*}{$\begin{array}{l}\text { Cowgill } \\
\text { and } \\
\text { Drab- } \\
\text { kin's } \\
\text { BSA for- } \\
\text { mula }\end{array}$} & \multirow{2}{*}{$\begin{array}{c}\text { New } \\
\text { BSA } \\
\text { for- } \\
\text { mula }\end{array}$} & \multicolumn{2}{|c|}{$\begin{array}{c}\text { Bleomycin } \\
\left(10 \mathrm{iu} / \mathrm{m}^{2}\right)\end{array}$} & \multicolumn{2}{|c|}{$\begin{array}{c}\text { Busulfan (4 } \\
\left.\mathrm{mg} / \mathrm{m}^{2}\right)\end{array}$} & \multicolumn{2}{|c|}{$\begin{array}{c}\text { Cisplatin (70 } \\
\left.\mathrm{mg} / \mathrm{m}^{2}\right)\end{array}$} & \multicolumn{2}{|c|}{$\begin{array}{c}\text { Cytarabine (100 } \\
\left.\mathrm{mg} / \mathrm{m}^{2}\right)\end{array}$} & \multicolumn{2}{|c|}{$\begin{array}{l}\text { Dacarbazine } \\
\left(200 \mathrm{mg} / \mathrm{m}^{2}\right)\end{array}$} & \multicolumn{2}{|c|}{$\begin{array}{c}\text { Dronabinol } \\
\left(5 \mathrm{mg} / \mathrm{m}^{2}\right)\end{array}$} & \multicolumn{2}{|c|}{$\begin{array}{l}\text { Lomustine } \\
\left(60 \mathrm{mg} / \mathrm{m}^{2}\right)\end{array}$} \\
\hline & & & & & & CDF & NF & CDF & NF & CDF & NF & CDF & NF & CDF & NF & CDF & NF & CDF & NF \\
\hline $\begin{array}{l}\text { Pomera- } \\
\text { nian }\end{array}$ & 5.0 & 0.60 & 13.89 & 0.30 & $0.25^{\mathrm{b}}$ & 3.0 & 2.5 & 1.2 & 1.0 & 21 & 17.5 & 30.0 & 25.0 & 60.0 & 50.0 & 1.5 & 1.25 & 18.0 & 15.0 \\
\hline $\begin{array}{c}\text { Australian } \\
\text { Silky Ter- } \\
\text { rier }\end{array}$ & 5.0 & 0.50 & 20.0 & 0.30 & $0.23^{b}$ & 3.0 & 2.3 & 1.2 & 0.92 & 21 & 16.1 & 30.0 & 23 & 60.0 & 46.0 & 1.5 & 1.15 & 18.0 & 13.8 \\
\hline $\begin{array}{l}\text { Affenpin- } \\
\text { scher }\end{array}$ & 3.6 & 0.76 & 6.23 & 0.24 & 0.24 & 2.4 & 2.4 & 0.96 & 0.96 & 16.8 & 16.8 & 24.0 & 24.0 & 48.0 & 48.0 & 1.2 & 1.2 & 14.4 & 14.4 \\
\hline $\begin{array}{l}\text { English } \\
\text { Toy Span- } \\
\text { iel }\end{array}$ & 5.4 & 0.50 & 21.6 & 0.31 & $0.24^{b}$ & 3.1 & 2.4 & 1.24 & 0.96 & 21.7 & 16.8 & 31.0 & 24.0 & 62.0 & 48.0 & 1.55 & 1.20 & 18.6 & 14.4 \\
\hline Toy Terrier & 2.5 & 0.50 & 10.0 & 0.19 & $0.16^{b}$ & 1.9 & 1.6 & 0.76 & 0.64 & 13.3 & 11.2 & 19.0 & 16.0 & 36.0 & 32.0 & 0.95 & 0.80 & 11.4 & 9.6 \\
\hline $\begin{array}{l}\text { Toy Fox } \\
\text { Terrier }\end{array}$ & 3.0 & 0.50 & 12.0 & 0.21 & $0.17^{\mathrm{b}}$ & 2.1 & 1.7 & 0.84 & 0.68 & 14.7 & 11.9 & 21.0 & 17.0 & 42.0 & 34.0 & 1.05 & 0.85 & 12.6 & 10.2 \\
\hline $\begin{array}{l}\text { Silky Ter- } \\
\text { rier }\end{array}$ & 5.0 & 0.45 & 24.69 & 0.30 & $0.21^{\mathrm{b}}$ & 3.0 & 2.1 & 1.20 & 0.84 & 21 & 14.7 & 30.0 & 21.0 & 60.0 & 42.0 & 1.5 & 1.05 & 18.0 & 12.6 \\
\hline $\begin{array}{l}\text { Brussels } \\
\text { Griffon }\end{array}$ & 4.5 & 0.45 & 22.22 & 0.28 & $0.20^{\mathrm{b}}$ & 2.8 & 2.0 & 1.12 & 0.80 & 19.6 & 19.6 & 28.0 & 20.0 & 56.0 & 40.0 & 1.4 & 1.0 & 16.8 & 12.0 \\
\hline $\begin{array}{l}\text { Maltese } \\
\quad(\mathrm{m})\end{array}$ & 5.0 & 0.50 & 20.0 & 0.30 & $0.23^{b}$ & 3.0 & 2.3 & 1.20 & 0.92 & 21.0 & 16.1 & 30.0 & 23.0 & 60.0 & 46.0 & 1.5 & 1.15 & 18.0 & 13.8 \\
\hline Maltese (f) & 4.0 & 0.46 & 18.90 & 0.25 & $0.19^{b}$ & 2.5 & 1.9 & 1.00 & 0.76 & 17.5 & 13.3 & 25.0 & 19.0 & 50.0 & 38.0 & 1.25 & 0.95 & 15.0 & 11.4 \\
\hline $\begin{array}{l}\text { Little Lion } \\
\text { Dog }\end{array}$ & 4.0 & 0.70 & 8.16 & 0.25 & $0.24^{b}$ & 2.5 & 2.4 & 1.00 & 0.96 & 17.5 & 16.8 & 25.0 & 24.0 & 50.0 & 48.0 & 1.25 & 1.20 & 15.0 & 14.4 \\
\hline Bichonfrise & 5.0 & 0.60 & 13.89 & 0.30 & $0.25^{\mathrm{b}}$ & 3.0 & 2.5 & 1.20 & 1.00 & 21 & 17.5 & 30.0 & 25.0 & 60.0 & 50.0 & 1.50 & 1.25 & 18.0 & 15.0 \\
\hline $\begin{array}{c}\text { Small } \\
\text { Continen- } \\
\text { tal Spaniel } \\
(\mathrm{m})\end{array}$ & 4.5 & 0.56 & 14.35 & 0.28 & $0.23^{b}$ & 2.8 & 2.3 & 0.96 & 0.92 & 19.6 & 16.1 & 28.0 & 23.0 & 56.0 & 46.0 & 1.40 & 0.92 & 16.8 & 13.8 \\
\hline $\begin{array}{c}\text { Small } \\
\text { Continen- } \\
\text { tal Spaniel } \\
\text { (f) }\end{array}$ & 1.5 & 0.50 & 6.0 & 0.13 & $0.21^{\mathrm{a}}$ & 1.3 & 2.1 & 0.52 & 0.84 & 9.1 & 14.7 & 13.0 & 21.0 & 26.0 & 42.0 & 0.65 & 1.05 & 7.8 & 12.6 \\
\hline Papillon & 4.5 & 0.56 & 14.35 & 0.28 & $0.23^{b}$ & 2.8 & 2.3 & 0.96 & 0.92 & 19.6 & 16.1 & 28.0 & 23.0 & 56.0 & 46.0 & 1.40 & 1.15 & 16.8 & 13.8 \\
\hline $\begin{array}{c}\text { Coton De } \\
\text { Tutear }(\mathrm{m})\end{array}$ & 4.0 & 0.62 & 10.41 & 0.25 & $0.23^{b}$ & 2.5 & 2.3 & 1.00 & 0.92 & 17.5 & 16.1 & 25.0 & 23.0 & 50.0 & 46.0 & 1.25 & 1.15 & 15.0 & 13.8 \\
\hline $\begin{array}{l}\text { Coton De } \\
\text { Tutear (f) }\end{array}$ & 3.5 & 0.56 & 11.16 & 0.23 & $0.20^{\mathrm{b}}$ & 2.3 & 2.0 & 0.96 & 0.80 & 16.1 & 14.0 & 23.0 & 20.0 & 46.0 & 40.0 & 1.15 & 1.00 & 13.8 & 12.0 \\
\hline $\begin{array}{l}\text { Mexican } \\
\text { Hairless } \\
\text { Dog }\end{array}$ & 13.6 & 1.00 & 13.60 & 0.58 & $0.56^{\mathrm{b}}$ & 5.8 & 5.6 & 2.32 & 2.24 & 40.6 & 39.2 & 58.0 & 56.0 & 116 & 114 & 2.9 & 2.8 & 34.8 & 33.6 \\
\hline Chihuahua & 2.6 & 0.44 & 13.43 & 0.19 & $0.15^{\mathrm{b}}$ & 1.9 & 1.5 & 0.76 & 0.60 & 13.3 & 10.5 & 19.0 & 15.0 & 38.0 & 30.0 & 0.95 & 0.75 & 11.4 & 9.0 \\
\hline $\begin{array}{l}\text { Chinese } \\
\text { Crested } \\
\text { Dog }\end{array}$ & 4.5 & 0.60 & 12.5 & 0.28 & $0.24^{\mathrm{b}}$ & 2.8 & 2.4 & 1.12 & 0.96 & 19.6 & 16.8 & 28.0 & 24.0 & 56.0 & 48 & 1.40 & 1.20 & 16.8 & 14.4 \\
\hline $\begin{array}{l}\text { Chinese } \\
\text { Imperial } \\
\text { Ch' In (Gi- } \\
\text { ant) }\end{array}$ & 7.0 & 0.50 & 28.0 & 0.37 & $0.27^{\mathrm{b}}$ & 3.7 & 2.7 & 1.48 & 1.08 & 25.9 & 18.9 & 37.0 & 27.0 & 74.0 & 54.0 & 1.85 & 1.35 & 22.2 & 16.2 \\
\hline $\begin{array}{c}\text { Chinese } \\
\text { Temple } \\
\text { Dog (Gi- } \\
\text { ant) }\end{array}$ & 9.0 & 0.72 & 17.36 & 0.44 & $0.38^{b}$ & 4.4 & 3.8 & 1.76 & 1.52 & 30.8 & 26.6 & 44.0 & 38.0 & 88.0 & 76.0 & 2.20 & 1.90 & 26.4 & 22.8 \\
\hline $\begin{array}{l}\text { Chinese } \\
\text { Impe- } \\
\text { rial Ch' In } \\
\text { (Classic) }\end{array}$ & 2.5 & 0.30 & 27.78 & 0.19 & 0.12 & 1.9 & 1.2 & 0.76 & 0.48 & 13.3 & 8.4 & 19.0 & 12.0 & 38.0 & 24.0 & 0.95 & 0.6 & 11.4 & 7.2 \\
\hline $\begin{array}{l}\text { Chinese } \\
\text { Impe- } \\
\text { rial Ch' In } \\
\text { (Miniature) }\end{array}$ & 1.8 & 0.20 & 45.00 & 0.15 & 0.08 & 1.5 & 0.8 & 0.60 & 0.32 & 10.5 & 5.6 & 15.0 & 8.0 & 30.0 & 16.0 & 0.75 & 0.4 & 9.0 & 4.8 \\
\hline $\begin{array}{l}\text { Chinese } \\
\text { Impe- } \\
\text { rial Ch' In } \\
\text { (Sleeve) }\end{array}$ & 0.7 & 0.16 & 27.3 & 0.08 & 0.04 & 0.8 & 0.4 & 0.32 & 0.16 & 5.6 & 2.8 & 8.0 & 4.0 & 16.0 & 8.0 & 0.40 & 0.20 & 4.8 & 2.4 \\
\hline
\end{tabular}


Citation: Saganuwan SA (2017) Derivation of a Unique Body Surface Area (Bsa) Formula for Calculation of Relatively Safe Doses of Dog and Human Anticancer Drugs. J Cancer Sci Ther 9: 690-704. doi:10.4172/1948-5956.1000493

\begin{tabular}{|c|c|c|c|c|c|c|c|c|c|c|c|c|c|c|c|c|c|c|c|}
\hline \begin{tabular}{|c|} 
Chinese \\
Temple \\
Dog (Clas- \\
sic)
\end{tabular} & 6.8 & 0.60 & 0.89 & 0.36 & 0.29 & 3.6 & 2.9 & 1.44 & 1.16 & 25.2 & 20.3 & 36.0 & 29.0 & 72.0 & 58.0 & 1.80 & 1.45 & 21.6 & 17.4 \\
\hline $\begin{array}{l}\text { Chinese } \\
\text { Temple } \\
\text { Dog (Min- } \\
\text { iature) }\end{array}$ & 1.8 & 0.26 & 26.63 & 0.15 & 0.09 & 1.5 & 0.9 & 0.6 & 0.36 & 10.5 & 6.3 & 16.0 & 9.0 & 30.0 & 18.0 & 0.75 & 0.45 & 9.0 & 5.4 \\
\hline $\begin{array}{l}\text { Chinese } \\
\text { Temple } \\
\text { Dog } \\
\text { (Sleeve) }\end{array}$ & 0.9 & 0.15 & 4.00 & 0.09 & 0.05 & 0.9 & 0.5 & 0.36 & 0.20 & 6.3 & 3.5 & 9.0 & 5.0 & 18.0 & 10.0 & 0.45 & 0.25 & 5.4 & 3.0 \\
\hline Pekingese & 4.5 & 0.90 & 5.56 & 0.28 & $0.29^{a}$ & 2.8 & 2.9 & 1.12 & 1.16 & 19.6 & 20.3 & 28.0 & 29.0 & 56.0 & 58.0 & 1.40 & 1.45 & 16.8 & 17.4 \\
\hline Shih-Tzu & 6.4 & 0.54 & 21.95 & 0.35 & $0.27^{b}$ & 3.5 & 2.7 & 1.40 & 1.08 & 24.5 & 18.9 & 35.0 & 27.0 & 70.0 & 54.0 & 1.75 & 1.35 & 21.0 & 16.2 \\
\hline $\begin{array}{c}\text { Lhasa } \\
\text { Apso }\end{array}$ & 6.8 & 0.56 & 21.68 & 0.36 & $0.28^{b}$ & 3.6 & 2.8 & 1.44 & 1.12 & 25.2 & 19.6 & 36.0 & 28.0 & 72.0 & 56.0 & 1.80 & 1.40 & 21.6 & 16.8 \\
\hline $\begin{array}{l}\text { Japanese } \\
\text { Spaniel } \\
\text { Chin }\end{array}$ & 3.2 & 0.60 & 8.89 & 0.22 & $0.20^{\mathrm{b}}$ & 2.2 & 2.0 & 0.88 & 0.80 & 15.4 & 14 & 22.0 & 20.0 & 44.0 & 40.0 & 1.10 & 1.00 & 13.2 & 12.0 \\
\hline $\begin{array}{c}\text { Yorkshire } \\
\text { Terrier }\end{array}$ & 3.5 & 0.46 & 16.54 & 0.23 & $0.18^{b}$ & 2.3 & 1.8 & 0.92 & 0.72 & 16.1 & 12.6 & 23.0 & 18.0 & 46.0 & 36.0 & 1.15 & 0.90 & 13.8 & 10.8 \\
\hline $\begin{array}{l}\text { Small Eng- } \\
\text { lish Terrier }\end{array}$ & 3.6 & 0.60 & 10.00 & 0.24 & $0.21^{b}$ & 2.4 & 2.1 & 0.96 & 0.84 & 16.8 & 14.7 & 24.0 & 21.0 & 48.0 & 42.0 & 1.20 & 1.05 & 14.4 & 12.6 \\
\hline $\begin{array}{c}\text { Australian } \\
\text { Terrier }\end{array}$ & 5.0 & 0.50 & 20.00 & 0.30 & $0.23^{\mathrm{b}}$ & 3.0 & 2.3 & 1.20 & 0.92 & 21 & 16.1 & 30.0 & 23.0 & 60.0 & 46.0 & 1.50 & 1.15 & 18.0 & 13.8 \\
\hline
\end{tabular}

Table 4: Body weight, height, body mass index and therapeutic doses of anticancer drugs for toys.

\begin{tabular}{|c|c|c|c|c|c|c|c|c|c|c|c|c|c|c|c|c|c|c|c|}
\hline \multirow[t]{2}{*}{ Breed } & \multirow[t]{2}{*}{$\begin{array}{c}\text { Body } \\
\text { weight } \\
\text { (kg) }\end{array}$} & \multirow[t]{2}{*}{$\begin{array}{l}\text { Height } \\
\text { x2 (m) }\end{array}$} & \multirow[t]{2}{*}{$\begin{array}{l}\text { Body } \\
\text { index }\end{array}$} & \multirow{2}{*}{$\begin{array}{c}\text { Cowgill } \\
\text { and Drab- } \\
\text { kin's BSA } \\
\text { formula }\end{array}$} & \multirow[t]{2}{*}{$\begin{array}{c}\text { New } \\
\text { BSA } \\
\text { formula }\end{array}$} & \multicolumn{2}{|c|}{$\begin{array}{l}\text { Carmustine } \\
\left(90 \mathrm{mg} / \mathrm{m}^{2}\right)\end{array}$} & \multicolumn{2}{|c|}{$\begin{array}{l}\text { 6-Mercaptopu- } \\
\text { rine }\left(50 \mathrm{mg} / \mathrm{m}^{2}\right)\end{array}$} & \multicolumn{2}{|c|}{$\begin{array}{l}\text { Fluorouracil } \\
\left(150 \mathrm{mg} / \mathrm{m}^{2}\right)\end{array}$} & \multicolumn{2}{|c|}{$\begin{array}{c}\text { Mitoxantrone } \\
\left(6 \mathrm{mg} / \mathrm{m}^{2}\right)\end{array}$} & \multicolumn{2}{|c|}{$\begin{array}{c}\text { Thioguanine } \\
\left(25 \mathrm{mg} / \mathrm{m}^{2}\right)\end{array}$} & \multicolumn{2}{|c|}{$\begin{array}{c}\text { Streptozoto- } \\
\text { cin } \\
\left(500 \mathrm{mg} / \mathrm{m}^{2}\right)\end{array}$} & \multicolumn{2}{|c|}{$\begin{array}{c}\text { Thiotepa } \\
\left(0.5 \mathrm{mg} / \mathrm{m}^{2}\right)\end{array}$} \\
\hline & & & & & & CDF & NF & CDF & NF & CDF & NF & CDF & NF & CDF & NF & CDF & NF & CDF & NF \\
\hline $\begin{array}{l}\text { Poodle (Stan- } \\
\text { dard) }\end{array}$ & 22.0 & 0.76 & 38.09 & 0.79 & 0.62 & 71.1 & 55.8 & 39.5 & 16.0 & 118.5 & 93.0 & 4.94 & 3.72 & 19.75 & 15.5 & 395 & 310 & 0.40 & 0.31 \\
\hline Great Spitz & 18.0 & 0.80 & 28.13 & 0.69 & 0.57 & 62.1 & 51.3 & 34.5 & 1.3 & 03.5 & 85.5 & 4.14 & 3.42 & 17.25 & 14.25 & 345 & 285 & 0.35 & 0.29 \\
\hline Small Spitz & 3.75 & 0.56 & 11.96 & 0.24 & 0.21 & 21.6 & 8.9 & 21.6 & 8.9 & 36.0 & 31.5 & 1.44 & 1.26 & 6.0 & 3.25 & 120 & 105 & 0.12 & 0.11 \\
\hline $\begin{array}{l}\text { Volpino Italiano } \\
\qquad(\mathrm{m})\end{array}$ & 4.5 & 0.60 & 12.5 & 0.28 & 0.24 & 25.2 & 21.6 & 14.0 & 12.0 & 42.0 & 36.0 & 1.68 & 1.44 & 7.0 & 6.0 & 140 & 120 & 0.14 & 0.12 \\
\hline $\begin{array}{l}\text { Volpino Italiano } \\
\text { (f) }\end{array}$ & 4.0 & 0.56 & 12.76 & 0.25 & 0.21 & 22.5 & 18.9 & 12.5 & 10.5 & 37.5 & 31.5 & 1.5 & 1.26 & 6.25 & 5.25 & 125 & 105 & 0.13 & 0.11 \\
\hline $\begin{array}{c}\text { Japanese Spitz } \\
(\mathrm{m})\end{array}$ & 11.0 & 0.80 & 17.19 & 0.50 & 0.44 & 45.0 & 39.6 & 25.0 & 22.0 & 75.0 & 66.0 & 3.00 & 2.64 & 12.5 & 11.0 & 250 & 220 & 0.25 & 0.22 \\
\hline $\begin{array}{l}\text { Japanese Spitz } \\
\text { (f) }\end{array}$ & 10.0 & 0.70 & 20.41 & 0.47 & 0.39 & 42.3 & 35.1 & 23.5 & 19.5 & 70.5 & 58.5 & 2.82 & 2.34 & 11.75 & 9.75 & 235 & 195 & 0.24 & 0.20 \\
\hline Harlekinpinscher & 12.0 & 0.70 & 24.49 & 0.53 & 0.43 & 47.7 & 8.7 & 26.5 & 21.5 & 79.5 & 64.5 & 3.18 & 2.58 & 13.25 & 10.75 & 265 & 215 & 0.27 & 0.22 \\
\hline Dalmatian (m) & 27.0 & 1.20 & 18.75 & 0.91 & 0.88 & 81.9 & 79.2 & 45.5 & 44.0 & 136.5 & 132.0 & 5.46 & 5.28 & 22.75 & 22.0 & 455 & 440 & 0.46 & 0.44 \\
\hline Dalmatian (f) & 25.0 & 1.10 & 20.66 & 0.86 & 0.80 & 77.4 & 72.0 & 77.4 & 40 & 129.0 & 120 & 5.16 & 4.80 & 21.5 & 20.0 & 430 & 400 & 0.43 & 0.40 \\
\hline Kromfohrlander & 12.0 & 0.92 & 14.18 & 0.53 & 0.50 & 47.7 & 45.0 & 26.5 & 25 & 79.5 & 25.0 & 3.18 & 3.0 & 13.25 & 12.5 & 265 & 250 & 0.27 & 0.25 \\
\hline Pinscher & 8.0 & 0.96 & 8.68 & 0.40 & 0.41 & 36.0 & 36.9 & 20 & 20.5 & 20.0 & 20.5 & 2.4 & 2.41 & 10.0 & 10.25 & 200 & 205 & 0.20 & 0.21 \\
\hline Zweraschnauzer & 7.3 & 0.70 & 14.90 & 0.38 & 0.33 & 34.2 & 29.7 & 19.0 & 16.5 & 19.0 & 16.5 & 2.28 & 1.98 & 9.5 & 8.25 & 190 & 165 & 0.19 & 0.17 \\
\hline $\begin{array}{l}\text { Schipperkee } \\
\text { (large) }\end{array}$ & 9.0 & 0.66 & 20.66 & 0.44 & 0.36 & 39.6 & 32.4 & 22.0 & 18.0 & 22.0 & 18.0 & 2.64 & 2.16 & 11.0 & 9.0 & 220 & 180 & 0.22 & 0.18 \\
\hline $\begin{array}{l}\text { Belgian Griffon } \\
\text { (small) }\end{array}$ & 3.0 & 0.81 & 4.57 & 0.21 & 0.22 & 18.9 & 19.8 & 10.5 & 11.0 & 10.5 & 33.0 & 1.26 & 1.32 & 5.25 & 5.5 & 105 & 110 & 0.11 & 0.11 \\
\hline $\begin{array}{c}\text { Piccolo Braban- } \\
\text { tino }\end{array}$ & 4.5 & 0.60 & 12.5 & 0.28 & $0.24^{b}$ & 25.2 & 21.6 & 14.0 & 12.0 & 42.0 & 36.0 & 1.68 & 1.44 & 7.0 & 6.0 & 140 & 120 & 0.14 & 0.12 \\
\hline $\begin{array}{l}\text { English Bulldog } \\
(\mathrm{m})\end{array}$ & 25.0 & 1.00 & 25.0 & 0.86 & $0.77^{\mathrm{b}}$ & 77.4 & 69.3 & 43.0 & 38.5 & 129 & 115.5 & 5.16 & 4.62 & 21.5 & 19.25 & 430 & 385 & 0.43 & 0.39 \\
\hline $\begin{array}{l}\text { English Bulldog } \\
\text { (f) }\end{array}$ & 23.0 & 0.75 & 4.89 & 0.82 & 0.63 & 73.8 & 56.7 & 41 & 31.5 & 41.0 & 31.5 & 4.92 & 3.78 & 20.5 & 15.75 & 410 & 315 & 0.41 & 0.32 \\
\hline French Bulldog & 12.0 & 0.60 & 33.33 & 0.53 & 0.40 & 47.7 & 36.0 & 26.5 & 20.0 & 26.5 & 20.0 & 3.18 & 2.4 & 13.25 & 10.0 & 265 & 200 & 0.27 & 0.20 \\
\hline Bolognese (m) & 4.5 & 0.60 & 12.5 & 0.28 & $0.24^{b}$ & 25.2 & 21.6 & 14.0 & 12.0 & 42.0 & 36.0 & 1.68 & 1.44 & 7.0 & 6.0 & 140 & 120 & 0.14 & 0.12 \\
\hline Bolognese (f) & 4.0 & 0.56 & 12.76 & 0.25 & 0.21 & 22.5 & 18.9 & 12.5 & 10.5 & 12.5 & 10.5 & 1.5 & 1.26 & 6.25 & 5.25 & 125 & 105 & 0.13 & 0.11 \\
\hline Bichon Avanese & 6.0 & 0.50 & 24.0 & 0.33 & 0.25 & 29.7 & 22.5 & 16.5 & 12.5 & 16.5 & 12.5 & 1.98 & 1.5 & 8.25 & 6.25 & 165 & 125 & 0.17 & 0.13 \\
\hline Cane Nudo & 8.0 & 0.80 & 12.5 & 0.40 & 0.37 & 26.0 & 33.3 & 20 & 18.5 & 20.0 & 18.5 & 2.4 & 2.22 & 10.0 & 9.25 & 200 & 185 & 0.20 & 0.19 \\
\hline $\begin{array}{c}\text { Schipperkee (me- } \\
\text { dium) }\end{array}$ & 5.0 & 0.56 & 15.94 & 0.30 & 0.24 & 27.0 & 21.6 & 15.0 & 12.0 & 45.0 & 36.0 & 1.80 & 1.44 & 7.5 & 6.0 & 150 & 120 & 1.15 & 0.12 \\
\hline
\end{tabular}


Citation: Saganuwan SA (2017) Derivation of a Unique Body Surface Area (Bsa) Formula for Calculation of Relatively Safe Doses of Dog and Human Anticancer Drugs. J Cancer Sci Ther 9: 690-704. doi:10.4172/1948-5956.1000493

\begin{tabular}{|c|c|c|c|c|c|c|c|c|c|c|c|c|c|c|c|c|c|c|c|}
\hline $\begin{array}{l}\text { Schipperkee } \\
\text { (miniature) }\end{array}$ & 3.0 & 0.50 & 12.00 & 0.21 & 0.17 & 18.9 & 15.3 & 10.5 & 8.5 & 31.5 & 25.5 & 1.26 & 1.02 & 5.25 & 4.25 & 105 & 85 & 0.11 & 0.09 \\
\hline $\begin{array}{l}\text { Belgian Griffon } \\
\quad \text { (large) }\end{array}$ & 11.0 & 0.81 & 16.76 & 0.46 & 0.44 & 41.4 & 39.6 & 23.0 & 22.0 & 69.0 & 66.0 & 2.76 & 2.64 & 11.5 & 11.0 & 230 & 220 & 0.23 & 0.22 \\
\hline $\begin{array}{l}\text { Poodle (minia- } \\
\text { ture) }\end{array}$ & 7.0 & 0.51 & 26.91 & 0.37 & 0.27 & 33.3 & 24.3 & 18.5 & 13.5 & 55.5 & 40.5 & 2.22 & 1.62 & 9.25 & 6.75 & 185 & 135 & 0.19 & 0.14 \\
\hline Chow Chow & 27.0 & 1.01 & 26.47 & 0.91 & $0.80^{\mathrm{b}}$ & 81.9 & 72.0 & 45.5 & 40.0 & 136.5 & 120.0 & 5.46 & 4.80 & 22.75 & 20.0 & 455 & 400 & 0.46 & 0.40 \\
\hline Pug & 8.0 & 0.56 & 25.51 & 0.40 & 0.31 & 36.0 & 27.9 & 20 & 15.5 & 20.0 & 15.5 & 2.4 & 1.86 & 10.0 & 7.75 & 200 & 155 & 0.2 & 0.16 \\
\hline Shar - Pei (m) & 25.0 & 1.02 & 24.03 & 0.86 & 0.71 & 77.4 & 69.3 & 43.0 & 38.5 & 43.0 & 38.5 & 5.16 & 4.62 & 21.5 & 19.25 & 430 & 305 & 0.43 & 0.39 \\
\hline Shar - Pei (f) & 20.0 & 0.92 & 23.63 & 0.74 & 0.65 & 66.6 & 58.5 & 37.0 & 32.5 & 37.0 & 32.5 & 4.44 & 3.90 & 18.5 & 16.25 & 370 & 325 & 0.37 & 0.33 \\
\hline Tibetan Spaniel & 6.8 & 1.02 & 6.54 & 0.36 & 0.39 & 32.4 & 35.1 & 18.0 & 19.5 & 18.0 & 19.5 & 2.16 & 2.34 & 9.00 & 9.75 & 180 & 195 & 0.18 & 0.20 \\
\hline $\begin{array}{l}\text { King Charles } \\
\text { Spaniel }\end{array}$ & 8.8 & 0.64 & 21.51 & 0.43 & 0.35 & 38.7 & 31.5 & 21.5 & 17.5 & 21.5 & 17.5 & 2.58 & 2.10 & 10.75 & 8.75 & 215 & 175 & 0.22 & 0.18 \\
\hline Telomian & 14.0 & 0.96 & 15.19 & 0.59 & 0.55 & 53.1 & 49.5 & 29.5 & 27.5 & 29.5 & 27.5 & 3.54 & 3.30 & 14.75 & 13.75 & 295 & 275 & 0.30 & 0.28 \\
\hline Shiba Inu & 20.0 & 0.82 & 29.74 & 0.74 & 0.61 & 66.6 & 54.9 & 37.0 & 30.5 & 37.0 & 30.5 & 4.44 & 3.66 & 18.5 & 15.25 & 370 & 305 & 0.37 & 0.31 \\
\hline
\end{tabular}

Table 5: Body weight, height, body mass index, body surface areas and therapeutic doses of anticancer drugs for companions.

\begin{tabular}{|c|c|c|c|c|c|c|c|c|c|c|c|c|c|}
\hline \multirow[t]{2}{*}{ Breed } & \multirow{2}{*}{$\begin{array}{l}\text { Body weight } \\
(\mathrm{kg})\end{array}$} & \multirow{2}{*}{$\begin{array}{l}\text { Height } \\
\text { x2 (m) }\end{array}$} & \multirow[t]{2}{*}{ Body index } & \multirow{2}{*}{$\begin{array}{l}\text { Cowgill and } \\
\text { Drabkin's } \\
\text { BSA formula }\end{array}$} & \multirow{2}{*}{$\begin{array}{l}\text { New BSA } \\
\text { formula }\end{array}$} & \multicolumn{2}{|c|}{$\begin{array}{l}\text { Vinblastine } \\
\left(0.7 \mathrm{mg} / \mathrm{m}^{2}\right)\end{array}$} & \multicolumn{2}{|c|}{$\begin{array}{l}\text { Predinisolone } \\
\left(40 \mathrm{mg} / \mathrm{m}^{2}\right)\end{array}$} & \multicolumn{2}{|c|}{$\begin{array}{c}\text { L-Asparaginase } \\
\left(10,000 \mathrm{iu} / \mathrm{m}^{2}\right)\end{array}$} & \multicolumn{2}{|c|}{$\begin{array}{l}\text { Ifosfamide } \\
\left(50 \mathrm{mg} / \mathrm{m}^{2}\right)\end{array}$} \\
\hline & & & & & & CDF & NF & $\mathrm{CDF}$ & NF & CDF & NF & CDF & NF \\
\hline Smooth Fox Terrier & 8.0 & 0.80 & 12.50 & 0.40 & $0.37^{\mathrm{b}}$ & 0.28 & 0.26 & 16.0 & 14.8 & 4000 & 3700 & 20.0 & 18.5 \\
\hline Wire Haired Fox Terrier & 8.0 & 0.79 & 12.82 & 0.40 & $0.37^{\mathrm{b}}$ & 0.28 & 0.26 & 16.0 & 14.8 & 4000 & 3700 & 20.0 & 18.5 \\
\hline Airedale Terrier & 20.0 & 1.18 & 14.36 & 0.74 & $0.74^{\mathrm{b}}$ & 0.52 & 0.52 & 29.6 & 29.6 & 7400 & 7400 & 37.0 & 37.0 \\
\hline Bedlington Terrier & 10.5 & 0.84 & 14.88 & 0.48 & $0.44^{b}$ & 0.34 & 31 & 19.2 & 17.6 & 4800 & 4400 & 24.0 & 22.0 \\
\hline Border Terrier** & 7.0 & 0.50 & 28.00 & 0.37 & $0.27^{b}$ & 0.26 & 0.19 & 14.8 & 10.8 & 3700 & 2700 & 18.5 & 13.5 \\
\hline Bull Terrier & 28.0 & 1.12 & 22.38 & 0.93 & $0.86^{b}$ & 0.44 & 0.60 & 37.2 & 34.4 & 9300 & 8600 & 46.5 & 43.0 \\
\hline Cairn Terrier & 6.3 & 0.60 & 17.50 & 0.34 & $0.28^{b}$ & 0.24 & 0.20 & 13.6 & 11.2 & 3400 & 2800 & 17.0 & 14.0 \\
\hline Dandie Diomont Terrier ${ }^{\star * *}$ & 8.0 & 0.50 & 32.0 & 0.40 & $0.29^{b}$ & 0.28 & 0.20 & 11.6 & 11.2 & 4000 & 2900 & 20.0 & 14.5 \\
\hline Irish Terrier & 12.3 & 0.92 & 14.53 & 0.54 & $0.50^{\mathrm{b}}$ & 0.38 & 0.35 & 21.6 & 20.0 & 5400 & 5000 & 27.0 & 25.0 \\
\hline Jack Russell Terrier ${ }^{* \star *}$ & 9.0 & 0.44 & 46.49 & 0.44 & $0.29^{b}$ & 0.31 & 0.20 & 17.6 & 11.60 & 4400 & 2900 & 22.0 & 14.5 \\
\hline Kerry Blue Terrier & 17.0 & 0.96 & 18.45 & 0.67 & $0.61^{\mathrm{b}}$ & 0.45 & 0.43 & 26.8 & 24.4 & 6700 & 6100 & 33.5 & 30.5 \\
\hline Lakeland Terrier (m) & 7.7 & 0.74 & 14.06 & 0.39 & $0.35^{b}$ & 0.27 & 0.25 & 15.6 & 14.0 & 3900 & 3500 & 19.5 & 14.5 \\
\hline Lakeland Terrier (f) & 6.8 & 0.71 & 13.49 & 0.36 & $0.32^{b}$ & 0.25 & 0.22 & 14.4 & 12.8 & 3600 & 3200 & 18.0 & 16.0 \\
\hline Manchester Terrier** & 7.7 & 0.50 & 27.2 & 0.39 & $0.29^{b}$ & 0.27 & 0.20 & 15.6 & 11.6 & 3900 & 2900 & 19.5 & 14.5 \\
\hline Norfolk Terrier & 5.4 & 0.50 & 21.60 & 0.31 & $0.24^{b}$ & 0.22 & 0.17 & 12.4 & 9.6 & 3100 & 2400 & 15.5 & 12.0 \\
\hline Norwich Terrier & 4.5 & 0.50 & 18.00 & 0.28 & $0.21^{\mathrm{b}}$ & 0.20 & 0.15 & 11.2 & 8.4 & 2800 & 2100 & 14.0 & 10.5 \\
\hline Scottish Terrier ${ }^{* * *}$ & 10.5 & 0.56 & 33.48 & 0.48 & $0.36^{\mathrm{b}}$ & 0.34 & 0.25 & 19.2 & 14.4 & 4800 & 3600 & 24.0 & 18.0 \\
\hline Sealyham Terrier $(\mathrm{m})$ & 9.0 & 0.60 & 25.00 & 0.44 & $0.34^{b}$ & 0.31 & 0.24 & 17.6 & 13.6 & 4400 & 3400 & 22.0 & 17.0 \\
\hline Sealyham Terrier (f) & 8.1 & 0.58 & 24.08 & 0.41 & $0.32^{b}$ & 0.29 & 0.22 & 16.4 & 12.8 & 4100 & 3200 & 20.5 & 16.0 \\
\hline Sky Terrier *** & 11.5 & 0.51 & 44.21 & 0.51 & $0.36^{b}$ & 0.36 & 0.75 & 20.4 & 14.4 & 5100 & 3600 & 20.5 & 16.0 \\
\hline $\begin{array}{c}\text { Soft-Coated Wheaten } \\
\text { Terrier }\end{array}$ & 15.8 & 0.90 & 19.51 & 0.64 & $0.57^{\mathrm{b}}$ & 0.45 & 0.40 & 25.6 & 22.8 & 6400 & 5700 & 32.0 & 28.5 \\
\hline $\begin{array}{l}\text { Staffordshire Bull Terrier } \\
(\mathrm{m})^{* *}\end{array}$ & 17.0 & 0.80 & 26.56 & 0.67 & $0.56^{b}$ & 0.47 & 0.39 & 26.8 & 22.4 & 6700 & 5600 & 33.5 & 28.0 \\
\hline $\begin{array}{l}\text { Staffordshire Bull Terrier } \\
(f)^{* *}\end{array}$ & 15.0 & 0.73 & 25.97 & 0.61 & $0.51^{\mathrm{b}}$ & 0.43 & 0.36 & 24.4 & 20.4 & 6100 & 5100 & 30.5 & 25.5 \\
\hline Welsh Terrier & 9.5 & 0. & 15.61 & 0 & $0.40^{\circ}$ & 0.32 & 0.28 & 18.0 & 16.0 & 4500 & 4000 & 22.5 & 20.0 \\
\hline $\begin{array}{l}\text { West Highland White } \\
\text { Terrier }^{* \star *}\end{array}$ & 10.0 & 0.56 & 31.89 & 0.47 & $0.35^{b}$ & 0.33 & 0.25 & 18.6 & 14.0 & 4700 & 3500 & 23.5 & 17.5 \\
\hline Glen of Imaal Terrier $(m)^{\star \star \star}$ & 16.0 & 0.72 & 30.86 & 0.64 & $0.51^{\mathrm{b}}$ & 0.45 & 0.36 & 25.6 & 20.4 & 6400 & 5100 & 32.0 & 25.5 \\
\hline Glen of Imaal Terrier $(\mathrm{f})^{* * *}$ & 14.0 & 0.66 & 32.14 & 0.59 & $0.45^{b}$ & 0.41 & 0.29 & 23.6 & 18.0 & 5900 & 4500 & 29.5 & 22.5 \\
\hline German hunting Terrier $(\mathrm{m})$ & 10.0 & 0.80 & 15.63 & 0.47 & $0.42^{b}$ & 0.33 & 0.29 & 18.8 & 16.8 & 4700 & 4200 & 23.5 & 21.0 \\
\hline German hunting Terrier (f) & 8.5 & 0.78 & 13.97 & 0.42 & $0.38^{b}$ & 0.29 & 0.27 & 16.8 & 15.2 & 4200 & 3800 & 21.0 & 19.0 \\
\hline Bohemian Terrier & 9.0 & 0.70 & 18.37 & 0.44 & $0.37^{\mathrm{b}}$ & 0.31 & 0.26 & 17.6 & 14.8 & 4400 & 3700 & 22.0 & 18.5 \\
\hline American Pit Bull Terrier (m) & 20.0 & 0.92 & 23.63 & 0.74 & $0.65^{b}$ & 0.52 & 0.46 & 29.6 & 26.0 & 7400 & 6500 & 37.0 & 32.5 \\
\hline $\begin{array}{l}\text { American Pit Bull Terrier } \\
(\mathrm{f})^{* *}\end{array}$ & 18.0 & 0.83 & 26.13 & 0.69 & $0.58^{b}$ & 0.48 & 0.41 & 27.6 & 23.2 & 6900 & 5800 & 34.5 & 29.0 \\
\hline Boston Terrier & 11.3 & 0.86 & 15.28 & 0.51 & $0.47^{b}$ & 0.36 & 0.34 & 20.4 & 18.8 & 5100 & 4700 & 25.5 & 23.5 \\
\hline Tibetan Terrier & 13.6 & 0.80 & 21.25 & 0.58 & $0.49^{b}$ & 0.41 & 0.34 & 23.2 & 19.6 & 5800 & 4900 & 29.0 & 24.5 \\
\hline
\end{tabular}

CDF: Cowgill and Drabkin's formula; NF: New Formula; m: Male; f: Female; ${ }^{* *}$ : Prone to overweight, BMl>25 kg/m² ${ }^{* * *}:$ Prone to obesity; BMl>30 kg/m²

Table 6: Body weight, height, body mass index, body surface areas and therapeutic doses of some anticancer drugs for terriers. 
Citation: Saganuwan SA (2017) Derivation of a Unique Body Surface Area (Bsa) Formula for Calculation of Relatively Safe Doses of Dog and Human Anticancer Drugs. J Cancer Sci Ther 9: 690-704. doi:10.4172/1948-5956.1000493

\begin{tabular}{|c|c|c|c|c|c|c|c|c|c|c|c|c|c|c|c|}
\hline \multirow[t]{2}{*}{ Breed } & \multirow{2}{*}{$\begin{array}{c}\text { Body } \\
\text { weight } \\
\text { (kg) }\end{array}$} & \multirow{2}{*}{$\begin{array}{l}\text { Height } \\
x 2(\mathrm{~m})\end{array}$} & \multirow{2}{*}{$\begin{array}{l}\text { Body } \\
\text { index }\end{array}$} & \multirow{2}{*}{$\begin{array}{l}\text { Cowgill and } \\
\text { Drabkin's } \\
\text { BSA formula }\end{array}$} & \multirow{2}{*}{$\begin{array}{c}\text { New BSA } \\
\text { formula }\end{array}$} & \multicolumn{2}{|c|}{$\begin{array}{l}\text { Methotrexate } \\
\left(5 \mathrm{mg} / \mathrm{m}^{2}\right)\end{array}$} & \multicolumn{2}{|c|}{$\begin{array}{c}\text { Dactinomycin } \\
\left(1 \mathrm{mg} / \mathrm{m}^{2}\right)\end{array}$} & \multicolumn{2}{|c|}{$\begin{array}{l}\text { Mechlorethamine } \\
\left(5 \mathrm{mg} / \mathrm{m}^{2}\right)\end{array}$} & \multicolumn{2}{|c|}{$\begin{array}{l}\text { Thio-TEPA } \\
\left(9 \mathrm{mg} / \mathrm{m}^{2}\right)\end{array}$} & \multicolumn{2}{|c|}{$\begin{array}{c}\text { 6-Mercaptopurine } \\
\left(50 \mathrm{mg} / \mathrm{m}^{2}\right)\end{array}$} \\
\hline & & & & & & $\mathrm{CDF}$ & NF & CDF & $\mathrm{NF}$ & CDF & NF & CDF & NF & CDF & $\mathrm{NF}$ \\
\hline $\begin{array}{l}\text { Shorthaired Dachshund } \\
\text { (Normal) }\end{array}$ & 9.0 & 0.44 & 46.49 & 0.44 & 0.29 & 2.20 & 1.45 & 0.44 & 0.29 & 2.20 & 1.45 & 3.96 & 2.61 & 22.0 & 14.5 \\
\hline Longhaired Dachshund $^{\star \star}$ & 10.6 & 0.42 & 52.35 & 0.49 & 0.31 & 2.45 & 1.55 & 0.49 & 0.31 & 2.45 & 1.55 & 4.41 & 2.79 & 24.5 & 15.5 \\
\hline Wirehaired Dachshund ${ }^{\star \star}$ & 8.3 & 0.40 & 51.9 & 0.41 & 0.26 & 2.05 & 1.30 & 0.41 & 0.26 & 2.05 & 1.30 & 3.69 & 2.34 & 20.5 & 13.0 \\
\hline Hanover Hound $(m)^{\star *}$ & 45.0 & 1.14 & 34.68 & 1.28 & 1.12 & 6.40 & 5.60 & 1.28 & 1.12 & 6.40 & 5.60 & 11.52 & 10.08 & 64.0 & 56.0 \\
\hline Hanover Hound $(f)^{\star *}$ & 43.0 & 1.04 & 39.79 & 1.23 & 1.04 & 6.15 & 5.20 & 1.23 & 1.04 & 6.15 & 5.20 & 11.07 & 9.36 & 61.5 & 52.0 \\
\hline Bloodhound $(m)^{* * *}$ & 48.0 & 1.34 & 26.73 & 1.33 & 1.26 & 6.65 & 6.30 & 1.33 & 1.26 & 6.65 & 6.30 & 11.97 & 11.34 & 66.5 & 63.0 \\
\hline Bloodhound (f) ${ }^{* * \star}$ & 40.0 & 1.20 & 27.78 & 1.18 & 1.08 & 5.90 & 5.40 & 1.18 & 1.08 & 5.90 & 5.40 & 10.62 & 9.72 & 59.0 & 54.0 \\
\hline Kardian Bear Dog* & 22.0 & 1.20 & 15.28 & 0.79 & 0.79 & 3.95 & 3.95 & 0.79 & 0.79 & 3.95 & 3.95 & 7.11 & 7.11 & 39.5 & 39.5 \\
\hline Grand Bleu DeGaslogne & 35.0 & 1.40 & 17.86 & 1.08 & 1.09 & 5.40 & 5.45 & 1.08 & 1.09 & 5.40 & 5.45 & 9.72 & 9.81 & 54.0 & 54.5 \\
\hline $\begin{array}{l}\text { Grand Gascon Saintongeris } \\
(\mathrm{m})\end{array}$ & 32.0 & 1.41 & 6.10 & 1.02 & 1.05 & 5.10 & 5.25 & 1.02 & 1.05 & 5.10 & 5.25 & 9.18 & 9.45 & 51.0 & 52.5 \\
\hline $\begin{array}{l}\text { Grand Gascon Saintongeris } \\
\text { (f) }\end{array}$ & 30.0 & 1.30 & 17.75 & 0.98 & 0.83 & 4.90 & 4.85 & 0.98 & 0.97 & 4.90 & 4.85 & 8.82 & 8.73 & 49.0 & 48.5 \\
\hline Poitevin & 30.0 & 1.40 & .31 & 0.98 & 1.01 & 4.90 & 5.05 & 0.98 & 1.01 & 4.90 & 5.05 & 8.82 & 9.09 & 49.0 & 50.5 \\
\hline Billy (m) & 30.0 & 1.32 & 17.22 & 0.98 & 0.98 & 4.90 & 4.90 & 0.98 & 0.98 & 4.90 & 4.90 & 8.82 & 8.82 & 49.0 & 49.0 \\
\hline Billy (f) & 25.0 & 1.16 & 18.58 & 0.86 & 0.83 & 4.30 & 4.15 & 0.86 & 0.83 & 4.30 & 4.15 & 7.74 & 7.47 & 43.0 & 41.5 \\
\hline Chen Francais & 30.0 & 1.44 & 14.47 & 0.98 & 1.02 & 4.90 & 5.10 & 0.98 & 1.02 & 4.90 & 5.10 & 8.82 & 9.18 & 49.0 & 51.0 \\
\hline Swedish Elkhound & 30.0 & 1.26 & 18.90 & 0.98 & 0.95 & 4.90 & 4.75 & 0.98 & 0.95 & 4.90 & 4.75 & 8.82 & 8.55 & 49.0 & 47.5 \\
\hline English Fox hound $(m)^{* *}$ & 63.0 & 1.26 & 39.68 & 1.60 & 1.41 & 8.0 & 7.05 & 1.60 & 1.41 & 8.0 & 7.05 & 14.4 & 12.69 & 80.0 & 70.5 \\
\hline English Fox hound (f) ${ }^{* *}$ & 60.0 & 1.22 & 40.42 & 1.55 & 1.35 & 7.75 & 6.75 & 1.55 & 1.35 & 7.75 & 6.75 & 13.95 & 12.15 & 77.5 & 67.5 \\
\hline American Fox hound $(m){ }^{* *}$ & 63.0 & 1.26 & 39.68 & 1.60 & 1.41 & 8.0 & 7.05 & 1.60 & 1.41 & 8.0 & 7.05 & 14.4 & 12.69 & 80.0 & 70.5 \\
\hline American Fox hound (f) ** & 56.0 & 1.22 & 37.72 & 1.48 & 1.30 & 7.40 & 6.50 & 1.48 & 1.30 & 7.4 & 6.50 & 13.32 & 11.7 & 74.0 & 65.0 \\
\hline Trigg Hound $(m)$ & 25.0 & 1.22 & 16.84 & 0.86 & 0.85 & 4.30 & 4.25 & 0.86 & 0.85 & 4.30 & 4.25 & 7.74 & 7.65 & 43.0 & 42.5 \\
\hline Trigg Hound (f) & 20.0 & 1.12 & 15.94 & 0.74 & 0.72 & 3.70 & 3.60 & 0.74 & 0.72 & 3.70 & 3.60 & 6.66 & 6.48 & 37.0 & 36.0 \\
\hline Plott Hound (m) & 29.0 & 1.28 & 17.70 & 0.95 & 0.94 & 4.75 & 4.70 & 0.95 & 0.94 & 4.75 & 4.70 & 8.55 & 8.46 & 47.5 & 47.0 \\
\hline $\begin{array}{l}\text { Shorthaired Dachshound } \\
\text { (Minature) })^{* \star}\end{array}$ & 4.0 & 0.24 & 69.44 & 0.25 & 0.14 & 1.25 & 0.70 & 0.25 & 0.14 & 1.25 & 0.70 & 2.25 & 1.26 & 12.5 & 7.0 \\
\hline $\begin{array}{l}\text { Shorthaired Dachshound } \\
\text { (Toy) })^{* \star}\end{array}$ & 3.5 & 0.22 & 72.31 & 0.23 & 0.12 & 1.15 & 0.60 & 0.23 & 0.12 & 1.15 & 0.60 & 1.15 & 0.60 & 11.5 & 6.0 \\
\hline Petit Bleu & 20.0 & 1.00 & 20.00 & 0.74 & 0.68 & 3.70 & 3.0 & 0.74 & 0.68 & 3.70 & 3.0 & 3.70 & 3.40 & 37.0 & 30.0 \\
\hline Plott Hound (f) & 25.0 & 1.22 & 16.84 & 0.86 & 0.85 & 4.30 & 4.25 & 0.86 & 0.85 & 4.30 & 4.25 & 7.74 & 7.65 & 43.0 & 42.5 \\
\hline $\begin{array}{c}\text { Dachsbracke (West Phalian } \\
\text { Basset) }{ }^{* *}\end{array}$ & 18.0 & 0.70 & 36.73 & 0.69 & 0.53 & 3.45 & 2.65 & 0.69 & 0.53 & 3.45 & 2.6 & 6.21 & 4.77 & 34.5 & 26.5 \\
\hline German Spaniel & 20.0 & 00 & 0.0 & 0.74 & 0.68 & 3.70 & 3.40 & 0.74 & 0.68 & 3.70 & 3.40 & 6.66 & 6.12 & 37.0 & 34.0 \\
\hline Tiroler Bracke & 22.0 & 0.96 & 23.87 & 0.79 & 0.70 & 3.95 & 3.50 & 0.79 & 0.70 & 3.95 & 3.50 & 7.11 & 6.30 & 39.5 & 35.0 \\
\hline Mountain Hound & 18.0 & 1.00 & 18.00 & 0.69 & 0.64 & 3.45 & 3.20 & 0.69 & 0.64 & 3.45 & 3.20 & 6.21 & 5.76 & 34.5 & 32.0 \\
\hline Finnish Spitz & 27.0 & 1.00 & 22.00 & 0.91 & 0.80 & 4.55 & 4.00 & 0.91 & 0.80 & 4.55 & 4.00 & 8.19 & 7.20 & 45.5 & 40.0 \\
\hline Finnish Hound $(m)$ & 25.0 & 1.22 & 16.84 & 0.86 & 0.85 & 4.30 & 4.25 & 0.86 & 0.85 & 4.30 & 4.25 & 7.74 & 7.65 & 43.0 & 42.5 \\
\hline Finnish Hound (f) & 23.0 & 1.16 & 17.09 & 0.82 & 0.79 & 4.10 & 3.95 & 0.82 & 0.79 & 4.10 & 3.95 & 7.38 & 7.11 & 41.0 & 39.5 \\
\hline Steinbrack $k^{* \star *}$ & 22.0 & 0.90 & 27.16 & 0.79 & 0.68 & 3.95 & 3.40 & 0.79 & 0.68 & 3.95 & 3.40 & 7.11 & 6.12 & 39.5 & 34.0 \\
\hline Ogar Polski (m) & 32.0 & 1.30 & 18.93 & 1.02 & 1.00 & 5.10 & 5.00 & 1.02 & 1.00 & 5.10 & 5.00 & 9.18 & 9.00 & 51.0 & 50.0 \\
\hline Ogar Polski (f) & 26.0 & 1.20 & 18.06 & 0.89 & 0.86 & 4.45 & 4.30 & 0.89 & 0.86 & 4.45 & 4.30 & 8.01 & 7.74 & 44.5 & 43.0 \\
\hline Austrian Hound & 22.0 & 1.02 & 21.15 & 0.79 & 0.72 & 3.95 & 3.40 & 0.79 & 0.72 & 3.95 & 3.60 & 7.11 & 6.48 & 39.5 & 36.0 \\
\hline Bavarian Mountain Hound ${ }^{* *}$ & 35.0 & 1.00 & 33.00 & 1.08 & 0.91 & 5.40 & 4.55 & 1.08 & 0.91 & 5.40 & 4.55 & 9.72 & 8.19 & 54.0 & 45.5 \\
\hline Petit Bleu De Gascogne & 20.0 & 1.40 & 10.20 & 0.74 & 0.81 & 3.70 & 4.05 & 0.74 & 0.81 & 3.70 & 4.05 & 6.66 & 7.29 & 37.0 & 40.5 \\
\hline Ariegeuis & 30.0 & 1.20 & 20.83 & 0.98 & 0.93 & 4.90 & 4.65 & 0.98 & 0.93 & 4.90 & 4.65 & 8.82 & 8.31 & 49.0 & 46.5 \\
\hline Basset Artesien Normand ${ }^{* *}$ & 15.0 & 0.72 & 28.94 & 0.61 & 0.49 & 3.05 & 2.45 & 0.61 & 0.49 & 3.05 & 2.45 & 5.49 & 4.41 & 30.5 & 24.5 \\
\hline Basset Bleu De Gascogne ${ }^{* *}$ & 18.0 & 0.76 & 31.16 & 0.69 & 0.56 & 3.45 & 2.80 & 0.69 & 0.56 & 3.45 & 2.80 & 6.21 & 5.04 & 34.5 & 28.0 \\
\hline Basset Griffon Vendeen ${ }^{\star \star *}$ & 20.0 & 0.82 & 29.74 & 0.74 & 0.61 & 3.70 & 3.05 & 0.74 & 0.61 & 3.70 & 3.05 & 6.66 & 5.49 & 37.0 & 30.5 \\
\hline $\begin{array}{l}\text { Basset Fauve De } \\
\text { Bretagne }^{* \star}\end{array}$ & 18.0 & 0.72 & 34.72 & 0.69 & 0.56 & 3.45 & 2.80 & 0.69 & 0.56 & 45 & 2.80 & 6.21 & 5.04 & 34.5 & 28.0 \\
\hline Griffon Nivernais & 25.0 & 1.20 & 17.36 & 0.86 & 0.84 & 4.30 & 4.20 & 0.86 & 0.84 & 4.30 & 4.20 & 7.74 & 7.56 & 43.0 & 42.0 \\
\hline Harrier ${ }^{* *}$ & 46.0 & 1.00 & 46.00 & 1.30 & 1.06 & 6.50 & 5.30 & 1.30 & 1.06 & 6.50 & 5.30 & 9.27 & 9.54 & 65.0 & 53.0 \\
\hline Beagle & 13.6 & 0.80 & 21.25 & & 0.49 & 2.90 & 2.45 & 0.58 & 0.49 & 2.90 & 2.45 & 5.22 & 4.41 & 29.0 & 24.5 \\
\hline Beagle Harrier & 20.0 & 0.96 & 21.70 & 0.74 & 0.67 & 3.70 & 3.35 & 0.74 & 0.67 & 3.70 & 3.35 & 6.66 & 6.03 & 37.0 & 33.5 \\
\hline Otter hound & 35.0 & 1.30 & 20.71 & 1.08 & 1.05 & 5.40 & 5.25 & 1.08 & 1.05 & 5.40 & 5.25 & 9.72 & 9.45 & 54.0 & 52.5 \\
\hline Basset Hound ${ }^{* *}$ & 23.0 & 0.71 & 45.63 & 0.82 & 0.61 & 4.10 & 3.05 & 0.82 & 0.61 & 4.10 & 3.05 & 7.38 & 5.49 & 41.0 & 30.5 \\
\hline Italian Hound (m) & 28.0 & 1.16 & 20.81 & 0.93 & 0.88 & 4.65 & 4.40 & 0.93 & 0.88 & 4.65 & 4.40 & 8.37 & 7.92 & 46.5 & 44.0 \\
\hline
\end{tabular}


Citation: Saganuwan SA (2017) Derivation of a Unique Body Surface Area (Bsa) Formula for Calculation of Relatively Safe Doses of Dog and Human Anticancer Drugs. J Cancer Sci Ther 9: 690-704. doi:10.4172/1948-5956.1000493

\begin{tabular}{|c|c|c|c|c|c|c|c|c|c|c|c|c|c|c|c|}
\hline Italian Hound (f) & 18.0 & 0.96 & 19.53 & 0.69 & 0.63 & 3.45 & 3.15 & 0.69 & 0.63 & 3.45 & 3.15 & 6.21 & 5.67 & 34.5 & 31.5 \\
\hline Cirneco Dell Etna $(m)$ & 12.0 & 1.12 & 9.57 & 0.53 & 0.55 & 2.65 & 3.30 & 0.53 & 0.55 & 2.65 & 3.30 & 4.77 & 4.95 & 26.5 & 33.0 \\
\hline $\begin{array}{l}\text { Dachsbrcke (Montano - } \\
\text { Alpino) }\end{array}$ & 10.0 & 0.84 & 14.17 & 0.47 & 0.43 & 2.35 & 2.15 & 0.47 & 0.43 & 2.35 & 2.15 & 4.25 & 3.87 & 23.5 & 21.5 \\
\hline Elizabeth Beagle ${ }^{\star \star \star}$ & 10.0 & 0.60 & 27.78 & 0.47 & 0.36 & 2.35 & 1.80 & 0.47 & 0.36 & 2.35 & 1.80 & 4.23 & 3.24 & 23.5 & 18.0 \\
\hline Cirneco Dell Etna (f) & 10.0 & 0.96 & 10.85 & 0.47 & 0.46 & 2.35 & 2.30 & 0.47 & 0.46 & 2.35 & 2.30 & 4.23 & 4.14 & 23.5 & 23.0 \\
\hline Podengo Portuguese Grade & 30.0 & 1.40 & 15.31 & 0.98 & 1.01 & 4.90 & 5.05 & 0.98 & 1.01 & 4.90 & 5.05 & 8.82 & 9.09 & 49.0 & 50.5 \\
\hline Podengo Portuguese Medio & 20.0 & 1.10 & 16.53 & 0.74 & 0.72 & 3.70 & 3.60 & 0.74 & 0.72 & 3.70 & 3.60 & 6.66 & 6.48 & 37.0 & 36.0 \\
\hline $\begin{array}{l}\text { Podengo Portuguese } \\
\text { Pequeno }\end{array}$ & 5.0 & 0.60 & 13.89 & 0.30 & 0.25 & 1.50 & 1.25 & 0.30 & 0.25 & 1.50 & 1.25 & 2.70 & 2.25 & 15.0 & 12.5 \\
\hline Hamitton Hound & 25.0 & 1.20 & 17.36 & 0.86 & 0.84 & 4.30 & 4.20 & 0.86 & 0.84 & 4.30 & 4.20 & 7.74 & 7.56 & 43.0 & 42.0 \\
\hline $\operatorname{Drever}(m)^{* * *}$ & 15.0 & 0.74 & 27.39 & 0.61 & 0.50 & 3.05 & 2.50 & 0.61 & 0.50 & 30.5 & 2.50 & 5.49 & 4.50 & 30.5 & 25.0 \\
\hline Drever $(\mathrm{f})^{\star *}$ & 14.0 & 0.64 & 34.18 & 0.59 & 0.45 & 2.95 & 2.25 & 0.59 & 0.45 & 2.95 & 2.25 & 5.31 & 4.05 & 29.5 & 22.5 \\
\hline Swiss Hound & 20.0 & 1.10 & 16.53 & 0.74 & 0.72 & 3.70 & 3.60 & 0.74 & 0.72 & 3.70 & 3.60 & 6.66 & 4.05 & 37.0 & 36.0 \\
\hline Jura Hound ${ }^{* *}$ & 20.0 & 0.80 & 31.25 & 0.74 & 0.61 & 3.70 & 3.05 & 0.74 & 0.61 & 3.70 & 3.05 & 6.66 & 5.49 & 37.0 & 30.5 \\
\hline Burnese Hound ${ }^{* * *}$ & 18.0 & 0.80 & 28.13 & 0.69 & 0.57 & 3.45 & 2.85 & 0.69 & 0.57 & 3.45 & 2.85 & 6.21 & 5.13 & 34.5 & 28.5 \\
\hline Curernese Hound ${ }^{* *}$ & 28.0 & 0.80 & 43.75 & 0.93 & 0.72 & 4.65 & 3.60 & 0.93 & 0.72 & 4.65 & 3.60 & 8.37 & 6.48 & 46.5 & 36.0 \\
\hline Small Swiss Hound ${ }^{* * *}$ & 15.0 & 0.76 & 26.0 & 0.61 & 0.51 & 3.05 & 2.55 & 0.61 & 0.51 & 3.05 & 2.55 & 5.49 & 4.59 & 30.5 & 25.5 \\
\hline $\begin{array}{l}\text { Smooth-Haired Istrian } \\
\text { Hound }^{\star \star}\end{array}$ & 20.0 & 1.16 & 14.86 & 0.74 & 0.74 & 3.70 & 3.70 & 0.74 & 0.74 & 3.70 & 3.70 & 6.66 & 6.66 & 37.0 & 37.0 \\
\hline Posavac & 18.0 & 1.16 & 13.38 & 0.69 & 0.70 & 3.45 & 3.50 & 0.69 & 0.70 & 3.45 & 3.50 & 6.21 & 6.30 & 34.5 & 35.0 \\
\hline Rhodesia Ridgeback $(\mathrm{m})$ & 33.9 & 1.32 & 19.46 & 1.06 & 1.04 & 5.30 & 5.20 & 1.06 & 1.04 & 5.30 & 5.20 & 9.54 & 9.36 & 53.0 & 52.0 \\
\hline Rhodesia Ridgeback (f) & 29.4 & 1.30 & 17.40 & 0.96 & 0.96 & 4.80 & 4.80 & 0.96 & 0.96 & 4.80 & 4.80 & 8.64 & 8.64 & 4.80 & 4.80 \\
\hline Basenji (m) & 10.8 & 0.85 & 14.95 & 0.49 & 0.45 & 2.45 & 2.25 & 0.49 & 0.45 & 2.45 & 2.25 & 4.41 & 4.05 & 24.5 & 22.5 \\
\hline Basenji (f) & 9.9 & 0.80 & 15.47 & 0.47 & 0.42 & 2.35 & 2.10 & 0.47 & 0.42 & 2.35 & 2.10 & 4.23 & 3.78 & 23.5 & 21.0 \\
\hline $\begin{array}{l}\text { Black and Tan Coon } \\
\text { Hound }^{* *}\end{array}$ & 64.0 & 1.36 & 34.60 & 1.62 & 1.48 & 8.10 & 7.40 & 1.62 & 1.48 & 8.10 & 7.40 & 14.58 & 13.32 & 81.0 & 74.0 \\
\hline Bluetick Coon hound $(m)^{*}$ & 36.0 & 1.38 & 18.90 & 1.10 & 1.10 & 5.50 & 5.50 & 1.10 & 1.10 & 5.50 & 5.50 & 9.90 & 9.90 & 55.0 & 55.0 \\
\hline Bluetick Coon hound (f) & 29.0 & 1.28 & 17.70 & 0.95 & 0.94 & 4.75 & 4.70 & 0.95 & 0.94 & 4.75 & 4.70 & 8.55 & 8.46 & 47.5 & 47.0 \\
\hline Redbone Coon hound (m) & 32.0 & 1.32 & 18.37 & 1.02 & 1.01 & 5.10 & 5.05 & 1.02 & 1.01 & 5.10 & 5.05 & 9.18 & 9.09 & 51.0 & 50.5 \\
\hline Redbone Coon hound (f) & 29.0 & 1.28 & 17.70 & 0.95 & 0.94 & 4.75 & 4.70 & 0.95 & 0.94 & 4.75 & 4.70 & 8.55 & 8.46 & 47.5 & 47.0 \\
\hline Redtick Coon hound $(\mathrm{m})^{\star}$ & 36.0 & 1.38 & 18.90 & 1.10 & 1.10 & 5.50 & 5.50 & 1.10 & 1.10 & 5.50 & 5.50 & 9.9 & 9.9 & 55.0 & 55.0 \\
\hline Redtick Coon hound (f) & 29.0 & 1.28 & 17.70 & 0.95 & 0.94 & 4.75 & 4.70 & 0.95 & 0.94 & 4.75 & 4.70 & 8.55 & 8.46 & 47.5 & 47.0 \\
\hline $\begin{array}{l}\text { Treeing Walker Coon hound } \\
\qquad(\mathrm{m})^{\star \star}\end{array}$ & 65.0 & 1.38 & 34.13 & 1.63 & 1.50 & 8.15 & 7.50 & 1.63 & 1.50 & 8.15 & 7.50 & 14.67 & 13.50 & 81.5 & 75.0 \\
\hline $\begin{array}{l}\text { Treeing Walker Coon hound } \\
\text { (f) }\end{array}$ & 34.0 & 1.28 & 20.75 & 1.06 & 1.03 & 5.30 & 5.15 & 1.06 & 1.03 & 5.30 & 5.15 & 19.54 & 9.27 & 53.0 & 51.5 \\
\hline $\begin{array}{l}\text { Tennessee Treeing Brindle } \\
(\mathrm{m})\end{array}$ & 23.0 & 1.22 & 15.45 & 0.82 & 0.81 & 4.10 & 4.05 & 0.82 & 0.81 & 4.10 & 4.05 & 7.38 & 7.29 & 41.0 & 40.5 \\
\hline $\begin{array}{l}\text { Tennessee Treeing Brindle } \\
\text { (f) }\end{array}$ & 18.0 & 1.12 & 14.35 & 0.69 & 0.68 & 3.45 & 3.40 & 0.69 & 0.68 & 3.45 & 3.40 & 6.21 & 6.12 & 34.5 & 34.0 \\
\hline Westphalian Basset** & 18.0 & 0.70 & 36.73 & 0.69 & 0.53 & 3.45 & 2.65 & 0.69 & 0.53 & 3.45 & 2.65 & 6.21 & 4.77 & 34.5 & 26.5 \\
\hline Bosnian Hound & 24.0 & 1.12 & 19.13 & 0.84 & 0.80 & 4.20 & 4.0 & 0.84 & 0.80 & 4.20 & 4.0 & 7.56 & 7.20 & 42.0 & 40.0 \\
\hline $\begin{array}{c}\text { Yugoslavian Mountain } \\
\text { hound }\end{array}$ & 25.0 & 1.10 & 20.66 & 0.86 & 0.81 & 4.20 & 4.05 & 0.86 & 0.81 & 4.30 & 4.05 & 7.74 & 7.29 & 43.0 & 40.5 \\
\hline Yugoslavian Tricolor hound & 25.0 & 1.10 & 20.66 & 0.86 & 0.81 & 4.30 & 4.05 & 0.86 & 0.81 & 4.30 & 4.05 & 7.74 & 7.29 & 43.0 & 40.5 \\
\hline Black Forest hound & 22.0 & 1.10 & 18.18 & 0.79 & 0.75 & 3.95 & 3.75 & 0.79 & 0.75 & 3.95 & 3.75 & 7.11 & 6.75 & 39.5 & 39.5 \\
\hline Grand Anglo-Francais & 32.0 & 1.36 & 17.30 & 1.02 & 1.03 & 1.10 & 1.15 & 1.02 & 1.03 & 1.10 & 1.15 & 9.18 & 9.27 & 37.5 & 37.5 \\
\hline Porcelaine (m) & 28.0 & 1.16 & 20.81 & 0.93 & 0.88 & 4.65 & 4.40 & 0.93 & 0.88 & 4.65 & 4.40 & 8.37 & 7.92 & 11.0 & 11.5 \\
\hline Porcelaine (f) & 25.0 & 1.12 & 19.93 & 0.86 & 0.81 & 4.30 & 4.05 & 0.86 & 0.81 & 4.30 & 4.05 & 7.74 & 7.29 & 46.5 & 44.0 \\
\hline Anglo-Francais Tricolor & 25.0 & 1.00 & 25.0 & 0.86 & 0.77 & 4.30 & 3.85 & 0.86 & 0.77 & 4.30 & 3.85 & 7.74 & 6.93 & 43.0 & 40.5 \\
\hline Petit Anglo-Francais & 20.0 & 0.90 & 24.69 & 0.74 & 0.64 & 3.70 & 3.20 & 0.74 & 0.64 & 3.70 & 3.20 & 6.66 & 5.76 & 43.0 & 38.5 \\
\hline Transylvanian Hound & 35.0 & 1.30 & 20.71 & 1.08 & 1.05 & 5.40 & 1.25 & 1.08 & 1.05 & 5.40 & 1.25 & 9.72 & 9.45 & 37.0 & 32.0 \\
\hline Small Gray Elk Dog ${ }^{\star * *}$ & 30.0 & 1.04 & 27.74 & 0.98 & 0.86 & 4.90 & 4.30 & 0.98 & 0.86 & 4.90 & 4.30 & 8.82 & 7.74 & 54.0 & 12.5 \\
\hline Balkan Hound $(m)$ & 20.0 & 1.03 & 18.85 & 0.74 & 0.69 & 3.70 & 3.45 & 0.74 & 0.69 & 3.70 & 3.45 & 6.66 & 6.21 & 49.0 & 43.0 \\
\hline Balkan Hound (f) & 20.0 & 1.04 & 18.49 & 0.74 & 0.70 & 3.70 & 3.50 & 0.74 & 0.70 & 3.70 & 3.50 & 6.66 & 6.30 & 37.0 & 34.5 \\
\hline Lunde hound $(\mathrm{m})$ & 6.0 & 0.72 & 11.57 & 0.33 & 0.30 & 1.65 & 1.50 & 0.33 & 0.30 & 1.65 & 1.50 & 2.97 & 2.70 & 37.0 & 35.0 \\
\hline Lunde hound (f) & 6.0 & 0.68 & 12.98 & 0.33 & 0.29 & 1.65 & 1.45 & 0.33 & 0.29 & 1.65 & 1.45 & 2.97 & 2.61 & 16.5 & 15.0 \\
\hline Norwegian Elk hound & 20.0 & 1.00 & 20.0 & 0.74 & 0.68 & 3.70 & 3.40 & 0.74 & 0.68 & 3.70 & 3.40 & 6.66 & 6.12 & 16.5 & 14.5 \\
\hline Schillerstorare $(\mathrm{m})$ & 24.0 & 1.14 & 18.47 & 0.84 & 0.80 & 4.20 & 4.00 & 0.84 & 0.80 & 4.20 & 4.00 & 7.56 & 7.20 & 37.0 & 34.0 \\
\hline Schillerstovare (f) & 18.0 & 1.06 & 16.02 & 0.69 & 0.66 & 3.45 & 3.30 & 0.69 & 0.66 & 3.45 & 3.30 & 6.21 & 5.94 & 42.0 & 40.0 \\
\hline Greek Hound (m) & 20.0 & 1.10 & 16.53 & 0.74 & 0.72 & 3.70 & 3.60 & 0.74 & 0.72 & 3.70 & 3.60 & 6.66 & 6.48 & 34.5 & 33.0 \\
\hline Greek Hound (f) & 17.0 & 1.06 & 15.13 & 0.67 & 0.64 & 3.45 & 3.20 & 0.67 & 0.64 & 3.35 & 3.20 & 6.03 & 5.76 & 37.0 & 36.0 \\
\hline
\end{tabular}


Citation: Saganuwan SA (2017) Derivation of a Unique Body Surface Area (Bsa) Formula for Calculation of Relatively Safe Doses of Dog and Human Anticancer Drugs. J Cancer Sci Ther 9: 690-704. doi:10.4172/1948-5956.1000493

\begin{tabular}{|c|c|c|c|c|c|c|c|c|c|c|c|c|c|c|c|}
\hline Smalendsstovare & 18.0 & 1.00 & 18.0 & 0.69 & 0.64 & 3.45 & 3.20 & 0.69 & 0.64 & 3.45 & 3.20 & 6.21 & 5.76 & 33.5 & 32.0 \\
\hline Hygenhund & 24.0 & 1.20 & 16.67 & 0.84 & 0.83 & 4.20 & 4.15 & 0.84 & 0.83 & 4.20 & 4.15 & 7.56 & 7.47 & 34.5 & 32.0 \\
\hline Haldenstover (m) & 25.0 & 1.10 & 20.66 & 0.76 & 0.81 & 4.30 & 4.05 & 0.86 & 0.81 & 4.30 & 4.05 & 7.74 & 7.29 & 42.0 & 41.5 \\
\hline Haldenstover (f) & 20.0 & 1.00 & 20.0 & 0.74 & 0.68 & 3.70 & 3.40 & 0.74 & 0.68 & 3.70 & 3.40 & 6.66 & 6.12 & 43.0 & 40.5 \\
\hline Dunker & 22.0 & 1.10 & 18.18 & 1.79 & 0.75 & 3.95 & 3.75 & 0.79 & 0.75 & 3.95 & 3.75 & 7.11 & 6.75 & 37.0 & 34.0 \\
\hline $\begin{array}{c}\text { Transylvanian (Short } \\
\text { legged)** }\end{array}$ & 35.0 & 1.00 & 35.00 & 1.08 & 0.91 & 5.4 & 4.55 & 1.08 & 0.91 & 5.4 & 4.55 & 9.72 & 8.19 & 39.5 & 37.5 \\
\hline $\begin{array}{l}\text { Norwegian Elkhound (Grey } \\
\text { male) }\end{array}$ & 20.0 & 1.02 & 19.22 & 0.74 & 0.69 & 3.70 & 3.45 & 0.74 & 0.69 & 3.70 & 3.45 & 6.66 & 6.21 & 54.0 & 45.5 \\
\hline $\begin{array}{l}\text { Norwegian Elkhound grey } \\
\quad \text { (female) }\end{array}$ & 20.0 & 0.98 & 20.52 & 0.74 & 0.67 & 3.70 & 3.35 & 0.74 & 0.67 & 3.70 & 3.35 & 6.66 & 6.03 & 37.0 & 34.5 \\
\hline Somercet Harrier & 18.0 & 1.10 & 14.88 & 0.69 & 0.68 & 3.45 & 3.40 & 0.69 & 0.68 & 3.45 & 3.40 & 6.21 & 6.12 & 37.0 & 33.5 \\
\hline Sabueso Espanol (m) & 25.0 & 1.12 & 19.93 & 0.86 & 0.81 & 4.30 & 4.05 & 0.86 & 0.81 & 4.30 & 4.05 & 7.74 & 7.29 & 34.5 & 34.0 \\
\hline Sabueso Espanol (f) & 25.0 & 1.04 & 23.11 & 0.86 & 0.78 & 4.30 & 3.90 & 0.86 & 0.78 & 4.30 & 3.90 & 7.74 & 7.02 & 43.0 & 40.5 \\
\hline Rastreador Brasileiro & 25.0 & 1.30 & 14.79 & 0.86 & 0.88 & 4.30 & 4.40 & 0.86 & 0.88 & 4.30 & 4.40 & 7.74 & 7.92 & 43.0 & 39.0 \\
\hline Levesque & 30.0 & 1.44 & 14.47 & 0.98 & 1.02 & 4.90 & 5.10 & 0.98 & 1.02 & 4.90 & 5.10 & 8.82 & 9.18 & 43.0 & 44.0 \\
\hline $\begin{array}{l}\text { Briquette Griffon Vendeen } \\
\qquad(\mathrm{m})\end{array}$ & 24.0 & 1.10 & 19.83 & 0.84 & 0.79 & 4.20 & 3.95 & 0.84 & 0.79 & 4.20 & 3.95 & 7.56 & 7.11 & 49.0 & 51.0 \\
\hline $\begin{array}{l}\text { Briquette Griffon Vendeen } \\
\text { (f) }\end{array}$ & 16.0 & 1.06 & 14.24 & 0.64 & 0.62 & 3.20 & 3.10 & 0.64 & 0.62 & 3.20 & 3.10 & 5.76 & 5.58 & 42.0 & 39.5 \\
\hline $\begin{array}{l}\text { Petit Griffon Bleu De } \\
\text { Gascogne }\end{array}$ & 15.0 & 1.04 & 13.87 & 0.61 & 0.60 & 3.05 & 3.00 & 0.61 & 0.60 & 3.05 & 3.00 & 5.49 & 5.40 & 32.0 & 31.5 \\
\hline $\begin{array}{l}\text { Griffon Fauve De Bretagne } \\
\qquad(\mathrm{m})\end{array}$ & 20.0 & 1.10 & 16.53 & 0.74 & 0.72 & 3.70 & 3.60 & 0.74 & 0.72 & 3.70 & 3.60 & 6.66 & 6.48 & 30.5 & 30.0 \\
\hline $\begin{array}{l}\text { Griffon Fauve De Bretagne } \\
\text { (f) }\end{array}$ & 20.0 & 1.04 & 18.49 & 0.74 & 0.70 & 3.70 & 3.50 & 0.74 & 0.70 & 3.70 & 3.50 & 6.66 & 6.30 & 37.0 & 36.0 \\
\hline Kurzhaar & 32.0 & 1.28 & 19.53 & 1.02 & 0.99 & 5.10 & 4.95 & 1.02 & 0.99 & 5.10 & 4.95 & 9.18 & 8.91 & 37.0 & 35.0 \\
\hline Drahthaar $(m)^{*}$ & 32.0 & 1.34 & 17.82 & 1.02 & 1.02 & 5.10 & 5.10 & 1.02 & 1.02 & 5.10 & 5.10 & 1.18 & 1.18 & 51.0 & 49.5 \\
\hline Drahthaar (f) & 27.0 & 1.24 & 17.56 & 0.91 & 0.89 & 4.55 & 4.45 & 0.91 & 0.89 & 4.55 & 4.45 & 8.19 & 8.01 & 51.0 & 51.0 \\
\hline Grosser Miinsterlainder & 20.0 & 1.24 & 13.01 & 0.74 & 0.76 & 3.70 & 3.80 & 0.74 & 0.76 & 3.70 & 3.80 & 6.66 & 6.84 & 45.5 & 44.5 \\
\hline Kleiner Miinsterlainder (m) & 15.0 & 1.12 & 11.96 & 0.61 & 0.62 & 3.05 & 3.10 & 0.61 & 0.62 & 3.05 & 3.10 & 5.49 & 5.58 & 37.0 & 38.0 \\
\hline Kleiner Miinsterlainder (f) & 15.0 & 1.04 & 13.87 & 0.61 & 0.60 & 3.05 & 3.00 & 0.61 & 0.60 & 5.05 & 3.00 & 5.49 & 5.40 & 30.5 & 31.0 \\
\hline German long-Haired Porita & 30.0 & 1.40 & 15.31 & 0.98 & 1.01 & 4.90 & 5.05 & 0.98 & 1.01 & 4.90 & 5.05 & 8.82 & 9.09 & 30.5 & 30.0 \\
\hline Weimaraner $(m)^{*}$ & 38.0 & 1.40 & 19.39 & 1.14 & 1.14 & 5.70 & 5.70 & 1.14 & 1.14 & 5.70 & 5.70 & 10.26 & 10.26 & 49.0 & 50.5 \\
\hline Weimaraner (f) & 35.0 & 1.30 & 20.71 & 1.08 & 1.05 & 5.40 & 5.25 & 1.08 & 1.05 & 5.40 & 5.25 & 9.72 & 9.45 & 57.0 & 57.0 \\
\hline Perdiguero De Burgos & 30.0 & 1.50 & 13.33 & 0.98 & 1.04 & 4.90 & 5.20 & 0.98 & 1.04 & 4.90 & 5.20 & 8.82 & 9.36 & 54.0 & 52.5 \\
\hline Podenco Ibicenco (m) & 22.5 & 1.32 & 12.91 & 0.80 & 0.84 & 4.00 & 4.20 & 0.80 & 0.84 & 4.00 & 4.20 & 7.20 & 7.56 & 49.0 & 52.0 \\
\hline Podenco Ibicenco (f) & 19.0 & 1.23 & 12.56 & 0.72 & 0.74 & 3.60 & 3.70 & 0.72 & 0.74 & 3.60 & 3.70 & 6.48 & 6.66 & 40.0 & 42.0 \\
\hline Braque Ariegeois * & 30.0 & 1.34 & 16.71 & 0.98 & 0.98 & 4.90 & 4.90 & 0.98 & 0.98 & 4.90 & 4.90 & 8.82 & 8.82 & 36.0 & 37.0 \\
\hline Braque D'Aurvergne $(\mathrm{m})$ & 28.0 & 1.26 & 17.64 & 0.93 & 0.92 & 4.65 & 4.600 & 0.93 & 0.92 & 4.65 & 4.60 & 8.37 & 8.28 & 49.0 & 49.0 \\
\hline Braque D' Aurvergne $(f)^{\star}$ & 22.0 & 1.20 & 15.28 & 0.79 & 0.79 & 3.95 & 3.95 & 0.79 & 0.79 & 3.95 & 3.95 & 7.11 & 7.11 & 46.5 & 46.0 \\
\hline French Pointer & 32.0 & 1.30 & 18.93 & 1.02 & 1.00 & 5.10 & 5.00 & 1.02 & 1.00 & 5.10 & 5.00 & 1.18 & 9.00 & 39.5 & 39.5 \\
\hline Barbet & 15.0 & 1.20 & 10.42 & 0.61 & 0.64 & 3.05 & 3.20 & 0.61 & 0.64 & 3.05 & 3.20 & 5.49 & 5.76 & 51.0 & 50.0 \\
\hline Vizsla & 28.0 & 1.24 & 18.21 & 0.93 & 0.91 & 4.65 & 4.55 & 0.93 & 0.91 & 4.65 & 4.55 & 8.37 & 8.19 & 30.5 & 32.0 \\
\hline Brittany Spaniel (m) & 18.0 & 1.10 & 14.88 & 0.69 & 0.68 & 3.45 & 3.40 & 0.69 & 0.68 & 3.45 & 3.40 & 6.21 & 6.12 & 46.5 & 45.5 \\
\hline Brittany Spaniel (f) & 15.0 & 0.98 & 15.62 & 0.61 & 0.58 & 3.05 & 2.90 & 0.61 & 0.58 & 3.05 & 2.90 & 5.49 & 5.22 & 34.5 & 34.0 \\
\hline French Spaniel $(\mathrm{m})$ & 25.0 & 1.20 & 17.36 & 0.86 & 0.84 & 4.30 & 4.20 & 0.86 & 0.84 & 4.30 & 4.20 & 7.74 & 7.56 & 30.5 & 29.0 \\
\hline French Spaniel (f) & 20.0 & 1.16 & 14.86 & 0.74 & 0.74 & 3.70 & 3.70 & 0.74 & 0.74 & 3.70 & 3.70 & 6.66 & 6.66 & 43.0 & 42.0 \\
\hline Picardy Spaniel & 20.0 & 1.20 & 13.89 & 0.74 & 0.75 & 3.70 & 3.75 & 0.74 & 0.75 & 3.70 & 3.75 & 6.66 & 6.75 & 37.0 & 37.0 \\
\hline Pont-Audemere Spaniel & 24.0 & 1.16 & 17.84 & 0.84 & 0.81 & 4.20 & 4.05 & 0.84 & 0.81 & 4.20 & 4.05 & 7.56 & 7.29 & 37.0 & 37.5 \\
\hline Soft-Coated Griffon (m) & 25.0 & 1.20 & 17.36 & 0.86 & 0.84 & 4.30 & 4.20 & 0.86 & 0.84 & 4.30 & 4.20 & 7.74 & 7.56 & 42.0 & 40.5 \\
\hline Soft-Coated Griffon (f) & 20.0 & 1.10 & 16.53 & 0.74 & 0.72 & 3.70 & 3.60 & 0.74 & 0.72 & 3.70 & 3.60 & 6.66 & 6.48 & 43.0 & 42.0 \\
\hline Korthals $(m)$ & 27.0 & 1.20 & 18.75 & 0.91 & 0.88 & 4.55 & 4.40 & 0.91 & 0.88 & 4.55 & 4.40 & 8.19 & 7.92 & 37.0 & 36.0 \\
\hline Korthals (f) & 23.0 & 1.10 & 19.01 & 0.82 & 0.77 & 4.10 & 3.85 & 0.82 & 0.77 & 4.10 & 3.85 & 7.38 & 6.93 & 45.5 & 44.0 \\
\hline Grand Griffon Vendien & 25.0 & 1.30 & 14.79 & 0.86 & 0.88 & 4.30 & 4.40 & 0.86 & 0.88 & 4.30 & 4.40 & 7.74 & 7.92 & 41.0 & 38.5 \\
\hline Briquette & 20.0 & 1.16 & 14.86 & 0.74 & 0.74 & 3.70 & 3.70 & 0.74 & 0.74 & 3.70 & 3.70 & 6.66 & 6.66 & 43.0 & 44.0 \\
\hline $\begin{array}{l}\text { Gammel Dansk Horsehead } \\
(\mathrm{m})\end{array}$ & 24.0 & 1.16 & 17.84 & 0.84 & 0.81 & 4.20 & 4.05 & 0.84 & 0.81 & 4.20 & 4.05 & 7.56 & 7.29 & 37.0 & 37.0 \\
\hline $\begin{array}{l}\text { Gammel Dansk Horsehead } \\
\text { (f) }\end{array}$ & 18.0 & 1.08 & 15.43 & 0.69 & 0.67 & 3.45 & 3.35 & 0.69 & 0.67 & 3.45 & 3.35 & 6.21 & 6.03 & 42.0 & 40.5 \\
\hline Wetter houn & 20.0 & 1.10 & 16.53 & 0.74 & 0.77 & 3.70 & 3.60 & 0.74 & 0.72 & 3.70 & 3.60 & 6.66 & 6.48 & 34.5 & 33.5 \\
\hline Staby-Houn & 20.0 & 1.00 & 20.00 & 0.74 & 0.68 & 3.70 & 3.40 & 0.74 & 0.68 & 3.70 & 3.40 & 6.66 & 6.12 & 37.0 & 36.0 \\
\hline Spinone Italiano (m) & 37.0 & 1.30 & 21.89 & 1.12 & 1.08 & 5.60 & 5.40 & 1.12 & 1.08 & 5.60 & 5.40 & 10.08 & 9.72 & 37.0 & 34.0 \\
\hline
\end{tabular}


Citation: Saganuwan SA (2017) Derivation of a Unique Body Surface Area (Bsa) Formula for Calculation of Relatively Safe Doses of Dog and Human Anticancer Drugs. J Cancer Sci Ther 9: 690-704. doi:10.4172/1948-5956.1000493

\begin{tabular}{|c|c|c|c|c|c|c|c|c|c|c|c|c|c|c|c|}
\hline Spinone Italiano (f) & 32.0 & 1.40 & 16.33 & 1.02 & 1.04 & 5.10 & 5.20 & 1.02 & 1.04 & 5.10 & 5.20 & 9.18 & 9.36 & 56.0 & 54.0 \\
\hline Italian Pointer & 40.0 & 1.34 & 22.28 & 1.81 & 1.15 & 5.90 & 5.75 & 1.18 & 1.15 & 5.90 & 5.95 & 10.62 & 10.35 & 51.0 & 52.0 \\
\hline Perdiguero Portuguese (m) & 27.0 & 1.12 & 21.52 & 0.91 & 0.85 & 4.55 & 4.25 & 0.91 & 0.85 & 4.55 & 4.25 & 8.19 & 7.65 & 59.0 & 57.5 \\
\hline Perdiguero Portuguese (f) & 24.0 & 1.04 & 22.19 & 0.84 & 0.77 & 4.20 & 3.85 & 0.84 & 0.77 & 4.20 & 3.85 & 7.56 & 6.93 & 45.5 & 42.5 \\
\hline Cesky Foursek & 34.0 & 1.32 & 19.51 & 1.06 & 1.04 & 5.30 & 5.20 & 1.06 & 1.04 & 5.30 & 5.20 & 9.54 & 9.36 & 42.0 & 38.5 \\
\hline Cesky Foursek & 28.0 & 1.24 & 19.51 & 0.93 & 0.91 & 4.65 & 4.55 & 0.93 & 0.91 & 4.65 & 4.55 & 8.37 & 8.19 & 53.0 & 52.0 \\
\hline Drentse Patrijshond & 20.0 & 1.30 & 11.83 & 0.74 & 0.78 & 3.70 & 3.90 & 0.74 & 0.78 & 3.70 & 3.90 & 6.66 & 7.62 & 46.5 & 45.5 \\
\hline Pointer (m) & 30.0 & 1.24 & 19.51 & 0.98 & 0.94 & 4.90 & 4.70 & 0.98 & 0.94 & 4.90 & 4.70 & 8.82 & 8.46 & 37.0 & 39.0 \\
\hline Pointer (f) & 20.0 & 1.20 & 13.89 & 0.74 & 0.75 & 3.70 & 3.75 & 0.74 & 0.75 & 3.70 & 3.75 & 6.66 & 6.75 & 49.0 & 47.0 \\
\hline English Setter $(m)$ & 32.0 & 1.24 & 20.81 & 1.02 & 0.98 & 5.10 & 4.90 & 1.02 & 0.98 & 5.10 & 4.90 & 9.18 & 8.82 & 37.0 & 37.5 \\
\hline English Setter (f) & 27.0 & 1.16 & 20.07 & 0.91 & 0.86 & 4.55 & 4.30 & 0.91 & 0.86 & 4.55 & 4.30 & 8.19 & 7.74 & 50.0 & 49.0 \\
\hline Irish Setter (m) & 22.5 & 1.24 & 14.63 & 0.80 & 0.81 & 4.00 & 4.55 & 0.80 & 0.81 & 4.00 & 4.95 & 7.20 & 7.29 & 45.5 & 43.0 \\
\hline Irish Setter (f) & 22.0 & 1.20 & 15.28 & 0.79 & 0.79 & 3.95 & 3.95 & 0.79 & 0.79 & 3.95 & 3.95 & 7.11 & 7.11 & 40.0 & 49.5 \\
\hline Gordon Setter (m) & 33.0 & 1.26 & 20.79 & 1.04 & 1.00 & 5.20 & 5.00 & 1.04 & 1.00 & 5.20 & 5.00 & 9.36 & 9.00 & 39.5 & 39.5 \\
\hline Gordon Setter (f) & 22.0 & 1.22 & 14.78 & 0.79 & 0.80 & 3.95 & 4.00 & 0.79 & 0.80 & 3.95 & 4.00 & 7.11 & 7.20 & 52.0 & 50.0 \\
\hline Braque De Boarbonnais & 26.0 & 1.10 & 21.49 & 0.89 & 0.82 & 4.45 & 4.10 & 0.89 & 0.82 & 4.45 & 4.10 & 8.01 & 7.38 & 39.5 & 40.0 \\
\hline Braque Dupuy (m) & 28.0 & 1.36 & 15.14 & 0.93 & 0.96 & 4.65 & 4.80 & 0.93 & 0.96 & 4.65 & 4.80 & 8.37 & 8.64 & 44.5 & 41.0 \\
\hline Braque Dupuy (f) & 22.0 & 1.32 & 12.63 & 0.79 & 0.83 & 3.95 & 4.15 & 0.76 & 0.83 & 3.95 & 4.15 & 7.11 & 7.47 & 46.5 & 48.0 \\
\hline Braque Saint-German & 26.0 & 1.24 & 16.91 & 0.89 & 0.88 & 4.45 & 4.40 & 0.89 & 0.88 & 4.45 & 4.40 & 8.01 & 7.92 & 39.5 & 41.5 \\
\hline Braque Saint-German & 18.0 & 1.18 & 12.93 & 0.69 & 0.70 & 3.45 & 3.50 & 0.69 & 0.70 & 3.45 & 3.50 & 6.21 & 6.30 & 44.5 & 44.0 \\
\hline Stichelhaar & 20.0 & 1.32 & 11.48 & 0.74 & 0.79 & 3.70 & 3.95 & 0.74 & 0.79 & 3.70 & 3.95 & 6.66 & 7.11 & 34.5 & 35.0 \\
\hline Pudel Pointer & 25.0 & 1.30 & 14.79 & 0.86 & 0.88 & 4.30 & 4.40 & 0.86 & 0.88 & 4.30 & 4.40 & 7.74 & 7.92 & 37.0 & 39.5 \\
\hline Curly-Coated Retriever & 36.2 & 1.32 & 20.78 & 1.11 & 1.08 & 5.55 & 5.40 & 1.11 & 1.08 & 5.55 & 5.40 & 9.99 & 9.72 & 43.0 & 44.0 \\
\hline Flat-Coated Retriever & 32.0 & 1.16 & 23.78 & 1.02 & 0.94 & 5.10 & 4.70 & 1.02 & 0.94 & 5.10 & 4.70 & 9.18 & 8.46 & 55.5 & 54.0 \\
\hline Golden Retriever $(\mathrm{m})$ & 34.0 & 1.22 & 22.84 & 1.06 & 1.00 & 5.30 & 5.00 & 1.06 & 1.00 & 5.30 & 5.00 & 8.54 & 9.00 & 51.0 & 47.0 \\
\hline Golden Retriever (f) & 32.0 & 1.14 & 24.62 & 1.02 & 0.94 & 5.10 & 4.70 & 1.02 & 0.94 & 5.10 & 4.70 & 9.18 & 8.46 & 53.0 & 50.0 \\
\hline Labrador Retriever $(\mathrm{m})^{* * *}$ & 36.0 & 1.14 & 27.70 & 1.10 & 1.00 & 5.50 & 5.00 & 1.10 & 1.00 & 5.50 & 5.00 & 9.90 & 9.00 & 51.0 & 47.0 \\
\hline Labrador Retriever (f) & 24.0 & 1.12 & 19.13 & 0.84 & 0.80 & 4.20 & 4.00 & 0.84 & 0.80 & 4.20 & 4.00 & 7.56 & 7.20 & 55.0 & 50.0 \\
\hline Chesapeake Retriever (m) & 34.0 & 1.32 & 19.51 & 1.06 & 1.04 & 5.30 & 5.20 & 1.06 & 1.04 & 5.30 & 5.20 & 9.54 & 9.36 & 42.0 & 40.0 \\
\hline Chesapeake Retriever (f) & 28.0 & 1.22 & 18.81 & 0.93 & 0.90 & 4.65 & 4.50 & 0.93 & 0.90 & 4.65 & 4.50 & 8.37 & 8.1 & 53.0 & 52.0 \\
\hline English Cocker Spaniel (m) & 14.5 & 0.82 & 21.56 & 0.60 & 0.52 & 3.00 & 2.06 & 0.60 & 0.52 & 3.00 & 2.60 & 5.40 & 4.68 & 46.5 & 45.0 \\
\hline English Cocker Spaniel (f) & 12.7 & 0.79 & 20.35 & 0.55 & 0.47 & 2.75 & 2.35 & 0.55 & 0.47 & 2.75 & 2.35 & 4.95 & 4.23 & 30.0 & 26.0 \\
\hline Cocker Spaniel $(\mathrm{m})$ & 12.7 & 0.76 & 21.99 & 0.55 & 0.46 & 2.75 & 2.30 & 0.55 & 0.46 & 2.75 & 2.30 & 4.95 & 4.14 & 27.5 & 23.5 \\
\hline Cocker Spaniel (f) & 10.9 & 0.74 & 19.91 & 0.50 & 0.42 & 2.50 & 2.10 & 0.50 & 0.42 & 2.50 & 2.10 & 4.50 & 3.78 & 27.5 & 23.0 \\
\hline Clumber Spaniel $^{\star \star}$ & 31.5 & 0.92 & 37.22 & 1.01 & 0.83 & 5.05 & 4.15 & 1.01 & 0.83 & 5.05 & 4.15 & 9.09 & 7.47 & 25.0 & 21.0 \\
\hline English Springer Spaniel & 24.0 & 1.00 & 24.00 & 0.84 & 0.75 & 4.20 & 3.75 & 0.84 & 0.75 & 4.20 & 3.75 & 7.56 & 6.75 & 50.5 & 41.5 \\
\hline Welsh Springer Spaniel ${ }^{\star \star *}$ & 20.25 & 0.86 & 27.38 & 0.75 & 0.63 & 3.75 & 3.15 & 0.75 & 0.63 & 3.75 & 3.15 & 6.75 & 5.67 & 42.0 & 37.5 \\
\hline Irish Water Spaniel $(\mathrm{m})$ & 27.22 & 1.16 & 20.23 & 0.91 & 0.87 & 4.55 & 4.35 & 0.91 & 0.87 & 4.55 & 4.35 & 8.19 & 7.83 & 37.5 & 31.5 \\
\hline Irish Water Spaniel (f) & 25.0 & 1.12 & 19.93 & 0.86 & 0.81 & 4.30 & 4.05 & 0.86 & 0.81 & 4.30 & 4.05 & 7.74 & 7.29 & 45.5 & 31.5 \\
\hline Sussex Spaniel ** & 20.0 & 0.80 & 31.25 & 0.74 & 0.61 & 3.70 & 3.05 & 0.74 & 0.61 & 3.70 & 3.05 & 6.66 & 5.49 & 43.0 & 40.5 \\
\hline Field Spaniel *** & 22.5 & 0.92 & 26.58 & 0.80 & 0.69 & 4.00 & 3.45 & 0.80 & 0.69 & 4.00 & 3.45 & 7.20 & 6.21 & 37.0 & 30.5 \\
\hline American Water Spaniel & 20.0 & 0.90 & 24.69 & 0.74 & 0.64 & 3.70 & 3.20 & 0.74 & 0.64 & 3.70 & 3.20 & 6.66 & 5.76 & 40.0 & 34.5 \\
\hline Boykin Spaniel & 17.0 & 0.86 & 22.99 & 0.67 & 0.58 & 3.35 & 2.90 & 0.67 & 0.58 & 3.35 & 2.90 & 6.03 & 5.22 & 37.0 & 32.0 \\
\hline
\end{tabular}

Table 7: Body weight, height, body mass index, body surface areas and therapeutic doses of anticancer drugs in hunting dogs.

\begin{tabular}{|c|c|c|c|c|c|c|c|c|c|c|c|c|c|c|c|}
\hline \multirow{2}{*}{$\begin{array}{c}\text { Breed } \\
\text { German Shepherd }(\mathrm{m})\end{array}$} & \multirow{2}{*}{$\begin{array}{c}\begin{array}{c}\text { Body } \\
\text { weight } \\
\text { (kg) }\end{array} \\
40.0 \\
\end{array}$} & \multirow{2}{*}{$\begin{array}{c}\begin{array}{c}\text { Height } \\
\mathbf{x}(\mathbf{m})\end{array} \\
1.30\end{array}$} & \multirow{2}{*}{\begin{tabular}{|l|}
$\begin{array}{l}\text { Body } \\
\text { Mass } \\
\text { index }\end{array}$ \\
23.67 \\
\end{tabular}} & \multirow{2}{*}{$\begin{array}{c}\begin{array}{c}\text { Cowgill and } \\
\text { Drabkin's BSA } \\
\text { formula }\end{array} \\
1.18\end{array}$} & \multirow{2}{*}{$\begin{array}{c}\begin{array}{c}\text { New BSA } \\
\text { formula }\end{array} \\
1.13\end{array}$} & \multicolumn{2}{|c|}{$\begin{array}{c}\text { Dacarbazine } \\
\left(200 \mathrm{mg} / \mathrm{m}^{2}\right)\end{array}$} & \multicolumn{2}{|c|}{$\begin{array}{l}\text { Asparaginase } \\
\left(30,000 \mathrm{iu} / \mathrm{m}^{2}\right)\end{array}$} & \multicolumn{2}{|c|}{$\begin{array}{c}\text { Dactinomycin } \\
\left(1.5 \mathrm{mg} / \mathrm{m}^{2}\right)\end{array}$} & \multicolumn{2}{|c|}{$\begin{array}{l}\text { Epirubicine } \\
\left(30 \mathrm{mg} / \mathrm{m}^{2}\right)\end{array}$} & \multicolumn{2}{|c|}{$\begin{array}{l}\text { Prednisolone } \\
\left(10 \mathrm{mg} / \mathrm{m}^{2}\right)\end{array}$} \\
\hline & & & & & & 236.0 & 0 & 35400 & 33900 & 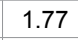 & 70 & 5.40 & 3.90 & 11.8 & 11.3 \\
\hline German Shepherd (f) & 35.0 & 1.20 & 24.30 & 1.08 & 1.01 & 216.0 & 202.0 & 32400 & 30 & 1.62 & 1.52 & 2.4 & 30.30 & 10.8 & 10.1 \\
\hline Belgian Shee & .0 & & 64 & 93 & & 6.0 & 184.0 & 7900 & 27600 & 1 & 1.38 & 7.9 & 27.6 & 3 & 2 \\
\hline Belgian Sh & & & & & & & 17 & 0 & & & & & & & 8 \\
\hline Belgian Tervuren & .0 & & 64 & & & 36.0 & 184.0 & 27900 & 27600 & & 38 & 27.9 & 27.6 & 3 & .2 \\
\hline Belgia & 0 & & 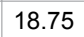 & & 88, & 82.0 & 17 & 2730 & 26400 & & 1.32 & 27 & 26.4 & & 8 \\
\hline Beauc & & & 86 & & & 216.0 & 218.0 & 32400 & 32700 & & 1.64 & 32.4 & 32.7 & 10.8 & 10.9 \\
\hline Briard $(m)^{\star * *}$ & 80 & 1.38 & .30 & 1.08 & 1.08 & 216.0 & 216.0 & 32400 & 32400 & 1.62 & 1.62 & 32.4 & 32.4 & 10.8 & 35400 \\
\hline Briard (f) & 35.0 & 1.20 & 1.36 & 1.08 & 1.04 & 216.0 & 208.0 & 32400 & 31200 & 1.62 & 1.56 & 32.4 & 31.2 & 10.8 & 10.4 \\
\hline $\begin{array}{c}\text { Smooth-Muzzled Pyrene } \\
\text { Sheepdog }\end{array}$ & 19 & 0.90 & 3.46 & 0.72 & 0 & 144.0 & 126.0 & 21600 & 18900 & 1.08 & 0.95 & 32.4 & 28.5 & 2 & 6.3 \\
\hline
\end{tabular}


Citation: Saganuwan SA (2017) Derivation of a Unique Body Surface Area (Bsa) Formula for Calculation of Relatively Safe Doses of Dog and Human Anticancer Drugs. J Cancer Sci Ther 9: 690-704. doi:10.4172/1948-5956.1000493

\begin{tabular}{|c|c|c|c|c|c|c|c|c|c|c|c|c|c|c|c|}
\hline Pyrenees Sheepdog & 20.0 & 1.00 & 20.00 & 0.74 & 0.68 & 148.0 & 136.0 & 22200 & 20400 & 1.11 & 1.02 & 33.3 & 30.6 & 7.4 & 6.8 \\
\hline Picardy Sheepdog & 32.0 & 1.30 & 18.93 & 1.02 & 1.00 & 204.0 & 200.0 & 30600 & 30000 & 1.53 & 1.50 & 45.9 & 45.0 & 10.2 & 10.0 \\
\hline Catalan Sheepdog (m) & 18.0 & 1.00 & 18.00 & 0.69 & 0.64 & 138.0 & 128.0 & 20700 & 19200 & 1.04 & 0.96 & 31.2 & 28.8 & 6.9 & 6.4 \\
\hline Catalan Sheepdog (f) & 16.0 & 0.96 & 17.36 & 0.64 & 0.59 & 128.0 & 118.0 & 19200 & 17700 & 0.96 & 0.89 & 28.8 & 26.7 & 6.4 & 5.9 \\
\hline Dutch Sheepdog $(\mathrm{m})$ & 30.0 & 1.26 & 18.90 & 0.98 & 0.95 & 196.0 & 190.0 & 29400 & 28500 & 1.47 & 1.43 & 44.10 & 42.9 & 9.8 & 9.5 \\
\hline Dutch Sheepdog (f) & 30.0 & 1.20 & 20.83 & 0.98 & 0.93 & 196.0 & 186.0 & 29400 & 27900 & 1.47 & 1.40 & 44.10 & 42.0 & 9.8 & 9.3 \\
\hline Komondor $(m)^{* *}$ & 59.0 & 1.30 & 34.91 & 1.53 & 1.38 & 306.0 & 276.0 & 45900 & 41400 & 2.30 & 2.07 & 69.0 & 62.1 & 15.3 & 13.8 \\
\hline Komondor $(\mathrm{f})$ ** & 50.0 & 1.28 & 30.52 & 1.37 & 1.26 & 274.0 & 252.0 & 41100 & 37800 & 2.06 & 1.89 & 61.8 & 56.7 & 13.7 & 12.6 \\
\hline Kuvasz & 50.0 & 1.50 & 22.22 & 1.37 & 1.37 & 274.0 & 274.0 & 41100 & 41100 & 2.06 & 2.06 & 61.8 & 61.8 & 13.7 & 13.7 \\
\hline Mudi & 13.0 & 0.94 & 14.71 & 0.56 & 0.52 & 112.0 & 104.0 & 16800 & 15600 & 0.84 & 0.78 & 25.2 & 23.4 & 5.6 & 5.2 \\
\hline Puli & 15.0 & 0.88 & 19.37 & 0.61 & 0.55 & 122.0 & 110.0 & 18300 & 16500 & 0.92 & 0.83 & 27.6 & 24.9 & 6.1 & 5.5 \\
\hline Pumi & 13.0 & 0.88 & 16.79 & 0.56 & 0.51 & 112.0 & 102.0 & 16800 & 15300 & 0.84 & 0.77 & 25.2 & 23.1 & 5.6 & 5.1 \\
\hline Anatolian Sheepdog $(\mathrm{m})^{\star *}$ & 68.0 & 1.04 & 62.87 & 1.68 & 1.33 & 336.0 & 266.0 & 50400 & 39900 & 2.25 & 2.00 & 50.4 & 39.9 & 25.2 & 20.0 \\
\hline Anatolian Sheepdog $(\mathrm{f}){ }^{* * *}$ & 59.0 & 1.42 & 29.26 & 1.53 & 1.45 & 306.0 & 290.0 & 45900 & 43500 & 2.30 & 2.18 & 45.9 & 43.5 & 23.0 & 21.8 \\
\hline $\operatorname{Bergamasco}(\mathrm{m})^{* \star *}$ & 38.0 & 1.20 & 26.39 & 1.14 & 1.05 & 228.0 & 210.0 & 34200 & 31500 & 1.71 & 1.58 & 34.2 & 31.5 & 17.1 & 15.8 \\
\hline Bergamasco (f) & 30.0 & 1.16 & 22.29 & 0.98 & 0.91 & 196.0 & 182.0 & 29400 & 27300 & 1.47 & 1.37 & 29.4 & 27.3 & 14.7 & 13.7 \\
\hline Maremma Sheepdog $(\mathrm{m})^{*}$ & 45.0 & 1.46 & 21.11 & 1.28 & 1.28 & 256.0 & 256.0 & 38400 & 38400 & 1.92 & 1.92 & 38.4 & 38.4 & 19.2 & 19.2 \\
\hline Maremma Sheepdog (f) & 40.0 & 1.36 & 21.63 & 1.18 & 1.15 & 236.0 & 230.0 & 35400 & 34500 & 1.77 & 1.73 & 35.4 & 34.5 & 17.7 & 17.3 \\
\hline Vala Sheepdog $(m)^{* * *}$ & 30.0 & 1.04 & 27.74 & 0.98 & 0.86 & 196.0 & 172.0 & 29400 & 25800 & 1.47 & 1.29 & 29.4 & 25.8 & 14.7 & 12.9 \\
\hline Vala Sheepdog $(\mathrm{f})^{\star *}$ & 30.0 & 0.92 & 35.44 & 0.98 & 0.81 & 196.0 & 162.0 & 29400 & 24300 & 1.47 & 1.23 & 29.4 & 24.3 & 14.7 & 12.3 \\
\hline Tatra Sheepdog (m) & 35.0 & 1.30 & 20.71 & 1.08 & 1.05 & 216.0 & 210.0 & 32400 & 31500 & 1.62 & 1.58 & 32.4 & 31.5 & 10.8 & 10.5 \\
\hline Tatra Sheepdog (f) & 30.0 & 1.20 & 20.83 & 0.98 & 0.93 & 196.0 & 186.0 & 29400 & 27900 & 1.47 & 1.40 & 29.4 & 27.9 & 9.8 & 9.3 \\
\hline Norsk Buhund ${ }^{* *}$ & 25.0 & 0.90 & 30.86 & 0.86 & 0.72 & 172.0 & 144.0 & 25800 & 21600 & 1.29 & 1.08 & 25.8 & 21.6 & 8.6 & 7.2 \\
\hline Swedish Shepherd & 14.0 & 0.80 & 21.88 & 0.59 & 0.50 & 118.0 & 100.0 & 17700 & 15000 & 0.89 & 0.75 & 17.7 & 15.0 & 5.9 & 5.0 \\
\hline Illyrian Sheepdog (m) & 35.0 & 1.20 & 24.31 & 1.08 & 1.01 & 216.0 & 202.0 & 32400 & 30300 & 1.62 & 1.52 & 32.4 & 30.3 & 10.8 & 10.1 \\
\hline Illyrian Sheepdog (f) & 25.0 & 1.10 & 20.66 & 0.86 & 0.81 & 172.0 & 162.0 & 25800 & 24300 & 1.29 & 1.22 & 25.8 & 24.3 & 8.6 & 8.1 \\
\hline Karst Sheepdog $(\mathrm{m})^{\star \star *}$ & 40.0 & 1.20 & 27.78 & 1.18 & 1.08 & 236.0 & 216.0 & 35400 & 32400 & 1.77 & 1.62 & 35.4 & 32.4 & 11.8 & 10.8 \\
\hline Karst Sheepdog (f) ** & 30.0 & 1.00 & 30.00 & 0.98 & 0.84 & 196.0 & 168.0 & 29400 & 25200 & 1.47 & 1.26 & 29.4 & 25.2 & 9.8 & 8.4 \\
\hline Bearded Collie & 30.0 & 1.12 & 23.92 & 0.98 & 0.90 & 196.0 & 180.0 & 29400 & 25200 & 1.47 & 1.35 & 29.4 & 27.0 & 9.8 & 8.4 \\
\hline Smooth Collie & 34.0 & 1.32 & 19.51 & 1.06 & 1.04 & 212.0 & 208.0 & 31800 & 31200 & 1.59 & 1.56 & 31.8 & 31.2 & 10.6 & 10.4 \\
\hline Rough Collie (m) & 34.0 & 1.32 & 19.51 & 1.06 & 1.04 & 212.0 & 208.0 & 31800 & 31200 & 1.59 & 1.56 & 31.8 & 31.2 & 10.6 & 10.4 \\
\hline Rough Collie (f) & 29.0 & 1.22 & 19.48 & 0.95 & 0.92 & 190.0 & 184.0 & 28500 & 27600 & 1.43 & 1.38 & 28.5 & 27.6 & 9.5 & 9.2 \\
\hline Border Collie (m) & 23.0 & 1.12 & 18.34 & 0.82 & 0.78 & 164.0 & 156.0 & 24600 & 23400 & 1.23 & 1.17 & 24.6 & 23.4 & 8.2 & 7.8 \\
\hline Border Collie (f) & 8.0 & 1.06 & 7.12 & 0.40 & 0.43 & 80.0 & 86.0 & 12000 & 12900 & 0.60 & 0.65 & 12.0 & 12.9 & 4.0 & 4.3 \\
\hline Bobtail & 30.0 & 1.12 & 23.92 & 0.98 & 0.90 & 196.0 & 180.0 & 29400 & 27000 & 1.47 & 1.35 & 29.4 & 27.0 & 9.8 & 9.0 \\
\hline Shetland Sheepdog & 7.0 & 0.82 & 10.41 & 0.37 & 0.35 & 74.0 & 70.0 & 11100 & 10500 & 0.56 & 0.53 & 11.1 & 10.5 & 3.7 & 3.5 \\
\hline Welsh Corgi** & 12.6 & 0.60 & 35.00 & 0.55 & 0.41 & 110.0 & 82.0 & 16500 & 12300 & 0.83 & 0.62 & 16.5 & 12.3 & 5.5 & 4.1 \\
\hline Rafeiro Do Alentejo & 50.0 & 1.50 & 22.22 & 1.37 & 1.37 & 274.0 & 274.0 & 41100 & 41100 & 2.06 & 2.06 & 41.1 & 41.1 & 13.7 & 13.7 \\
\hline Lapponian Herder (m) & 30.0 & 1.10 & 24.79 & 0.98 & 0.89 & 196.0 & 178.0 & 29400 & 26700 & 1.47 & 1.34 & 29.4 & 26.7 & 9.8 & 8.9 \\
\hline Lapponian Herder (f) ${ }^{\star *}$ & 30.0 & 0.98 & 31.24 & 0.90 & 0.83 & 180.0 & 166.0 & 27000 & 24900 & 1.35 & 1.25 & 27.0 & 24.9 & 9.0 & 8.3 \\
\hline Lapphund & 20.0 & 1.00 & 20.00 & 0.74 & 0.68 & 148.0 & 136.0 & 22200 & 20400 & 1.11 & 1.02 & 22.2 & 20.4 & 7.4 & 6.8 \\
\hline Slovak Tchouvatch $(\mathrm{m})$ & 45.0 & 1.40 & 22.96 & 1.12 & 1.25 & 224.0 & 250.0 & 33600 & 37500 & 1.68 & 1.88 & 33.6 & 37.5 & 11.2 & 12.5 \\
\hline Slovak Tchouvatch (f) & 40.0 & 1.30 & 23.67 & 1.18 & 1.13 & 236.0 & 226.0 & 35400 & 33900 & 1.77 & 1.70 & 35.4 & 33.9 & 11.8 & 11.3 \\
\hline Croatian Sheepdog & 20.0 & 1.00 & 20.00 & 0.74 & 0.68 & 148.0 & 136.0 & 22200 & 204.00 & 1.11 & 1.02 & 22.2 & 20.4 & 7.4 & 6.8 \\
\hline Iceland Dog (m) & 14.0 & 0.82 & 20.82 & 0.59 & 0.51 & 118.0 & 102.0 & 17700 & 15300 & 0.89 & 0.77 & 17.7 & 15.3 & 5.9 & 5.1 \\
\hline Iceland Dog (f) & 11.0 & 0.76 & 19.04 & 0.50 & 0.43 & 100.0 & 86.0 & 15000 & 12900 & 0.75 & 0.65 & 15.0 & 12.9 & 5.0 & 4.3 \\
\hline Australian Kelpie $(\mathrm{m})$ & 14.0 & 1.07 & 12.23 & 0.59 & 0.58 & 118.0 & 116.0 & 17700 & 17400 & 0.89 & 0.87 & 17.7 & 17.4 & 5.9 & 5.8 \\
\hline Australian Kelpie (f) & 11.0 & 0.96 & 11.94 & 0.50 & 0.49 & 100.0 & 98.0 & 15000 & 14700 & 0.75 & 0.74 & 15.0 & 14.7 & 5.0 & 4.9 \\
\hline Austrian Shepherd & 20.0 & 1.16 & 14.86 & 0.74 & 0.74 & 148.0 & 148.0 & 22200 & 22200 & 1.11 & 1.11 & 22.2 & 22.2 & 7.4 & 7.4 \\
\hline Austrian Shepherd & 18.0 & 1.06 & 16.02 & 0.69 & 0.66 & 138.0 & 132.0 & 20700 & 19800 & 1.04 & 0.99 & 20.7 & 19.8 & 6.9 & 6.6 \\
\hline Bourier Des Ardennes & 25.0 & 1.20 & 17.36 & 0.86 & 0.84 & 172.0 & 168.0 & 25800 & 25200 & 1.29 & 1.26 & 25.8 & 25.2 & 8.6 & 8.4 \\
\hline Cao DaSerra De Aires & 18.0 & 0.96 & 19.53 & 0.69 & 0.63 & 138.0 & 126.0 & 20700 & 18900 & 1.04 & 0.95 & 20.7 & 18.9 & 6.9 & 6.3 \\
\hline Bouvier Des Flanders & 40.0 & 1.36 & 21.63 & 1.18 & 1.15 & 236.0 & 230.0 & 35400 & 34500 & 1.77 & 1.73 & 35.4 & 34.5 & 11.8 & 11.5 \\
\hline Appenzel Mountain Dog & 25.0 & 1.16 & 18.58 & 0.86 & 0.83 & 172.0 & 166.0 & 25800 & 24900 & 1.29 & 1.25 & 25.8 & 24.9 & 8.6 & 8.3 \\
\hline Bernese Mountain Dog & 40.0 & 1.40 & 20.41 & 1.18 & 1.17 & 236.0 & 234.0 & 35400 & 35100 & 1.77 & 1.76 & 35.4 & 35.1 & 11.8 & 11.7 \\
\hline Great Swiss Mountain Dog** & 59.0 & 1.40 & 30.10 & 1.53 & 1.44 & 306.0 & 216.0 & 45900 & 43200 & 2.30 & 2.16 & 45.9 & 43.2 & 15.3 & 14.4 \\
\hline Entlebucher Sennehund ${ }^{* *}$ & 30.0 & 1.01 & 29.41 & 0.98 & 0.85 & 196.0 & 170.0 & 29400 & 25500 & 1.47 & 1.28 & 29.4 & 25.5 & 9.8 & 8.5 \\
\hline Austrian Cattle Dog ** & 23.0 & 0.96 & 35.81 & 1.04 & 0.87 & 208.0 & 174.0 & 31200 & 26100 & 1.56 & 1.31 & 31.2 & 26.1 & 10.4 & 8.7 \\
\hline Austrian Cattle Dog & 20.0 & 0.92 & 23.63 & 0.74 & 0.65 & 148.0 & 130.0 & 22200 & 19500 & 1.11 & 0.98 & 22.2 & 19.5 & 7.4 & 6.5 \\
\hline Catahoula leopard Dog $(\mathrm{m})$ & 36.0 & 1.28 & 21.97 & 1.10 & 1.06 & 220.0 & 212.0 & 33000 & 31800 & 1.65 & 1.59 & 33.0 & 31.8 & 11.0 & 10.6 \\
\hline Catahoula leopard Dog (f) & 32.0 & 1.18 & 22.98 & 1.02 & 0.95 & 204.0 & 190.0 & 30600 & 28500 & 1.53 & 1.43 & 30.6 & 28.5 & 10.2 & 9.5 \\
\hline
\end{tabular}


Citation: Saganuwan SA (2017) Derivation of a Unique Body Surface Area (Bsa) Formula for Calculation of Relatively Safe Doses of Dog and Human Anticancer Drugs. J Cancer Sci Ther 9: 690-704. doi:10.4172/1948-5956.1000493

\begin{tabular}{|c|c|c|c|c|c|c|c|c|c|c|c|c|c|c|c|}
\hline Standard Schnauzer & 15.0 & 1.00 & 15.00 & 0.61 & 0.58 & 122.0 & 116.0 & 18300 & 17400 & 0.92 & 0.87 & 18.3 & 17.4 & 6.1 & 5.8 \\
\hline Great Dane $(m)^{* * *}$ & 60.0 & 1.52 & 25.97 & 1.55 & 1.52 & 310.0 & 304.0 & 46500 & 45600 & 2.33 & 2.28 & 46.5 & 45.6 & 15.5 & 15.2 \\
\hline Great Dane (f) *** & 60.0 & 1.44 & 28.94 & 1.55 & 1.47 & 310.0 & 294.0 & 46500 & 44100 & 2.33 & 2.21 & 46.5 & 44.1 & 15.5 & 14.7 \\
\hline Tawny Great Dane ${ }^{\star *}$ & 54 & 1.22 & 36.28 & 1.44 & 1.28 & 288.0 & 256.0 & 43200 & 38400 & 2.16 & 1.92 & 43.2 & 38.4 & 14.4 & 12.8 \\
\hline Brindle Great Dane** & 58 & 1.30 & 34.32 & 1.51 & 1.32 & 302.0 & 284.0 & 45300 & 39600 & 2.27 & 1.98 & 45.3 & 39.6 & 15.1 & 13.2 \\
\hline Harlequin Great Dane* & 56 & 1.35 & 30.73 & 1.48 & 1.37 & 2960 & 274.0 & 44400 & 41100 & 2.22 & 2.06 & 44.4 & 41.1 & 14.8 & 13.7 \\
\hline Hovawart $(\mathrm{m})$ & 40.0 & 1.40 & 20.41 & 1.18 & 1.17 & 236.0 & 234.0 & 35400 & 35100 & 1.77 & 1.76 & 35.4 & 35.1 & 11.8 & 11.7 \\
\hline Hovawart (f) & 35.0 & 1.30 & 20.71 & 1.08 & 1.05 & 216.0 & 210.0 & 32400 & 31500 & 1.62 & 1.58 & 32.4 & 31.5 & 10.8 & 10.5 \\
\hline Napoleon Mastiff $(m)^{\star \star}$ & 70.0 & 1.50 & 31.11 & 1.72 & 1.63 & 344.0 & 326.0 & 51600 & 48900 & 2.58 & 2.45 & 51.6 & 48.9 & 17.2 & 16.3 \\
\hline Napoleon Mastiff (f) ** & 70.0 & 1.40 & 35.71 & 1.72 & 1.58 & 344.0 & 316.0 & 51600 & 47400 & 2.58 & 2.37 & 51.6 & 47.4 & 17.2 & 15.8 \\
\hline French Mastiff $(\mathrm{m})$ & 50.0 & 1.60 & 19.53 & 1.37 & 1.42 & 274.0 & 284.0 & 41100 & 42600 & 2.06 & 2.13 & 41.1 & 42.6 & 13.7 & 14.2 \\
\hline French Mastiff (f) & 50.0 & 1.44 & 24.11 & 1.37 & 1.34 & 274.0 & 268.0 & 41100 & 40200 & 2.06 & 2.01 & 41.1 & 40.2 & 13.7 & 13.4 \\
\hline Old English Mastiff $(m){ }^{* *}$ & 86.0 & 1.50 & 38.22 & 1.97 & 1.82 & 394.0 & 364.0 & 59100 & 54600 & 2.96 & 2.73 & 59.1 & 54.6 & 19.7 & 18.2 \\
\hline Old English Mastiff (f) ** & 79.0 & 1.40 & 40.31 & 1.86 & 1.68 & 372.0 & 336.0 & 55800 & 50400 & 2.79 & 2.52 & 55.8 & 50.4 & 18.6 & 16.8 \\
\hline Bullmastiff $(m)^{* *}$ & 58.0 & 1.37 & 30.90 & 1.51 & 1.41 & 302.0 & 282.0 & 45300 & 42300 & 2.27 & 2.12 & 45.3 & 42.3 & 15.1 & 14.1 \\
\hline Bullmastiff $(f)$ ** & 54.0 & 1.32 & 30.99 & 1.44 & 1.33 & 288.0 & 266.0 & 43200 & 39910 & 2.16 & 2.00 & 4.32 & 39.9 & 14.4 & 13.3 \\
\hline Rottweiler $* * *$ & 50.0 & 1.36 & 27.03 & 1.37 & 1.30 & 274.0 & 195.0 & 30600 & 29700 & 2.06 & 1.95 & 41.1 & 39.0 & 13.7 & 13.0 \\
\hline Boxer (m) & 32.0 & 1.26 & 20.16 & 1.02 & 0.99 & 204.0 & 198.0 & 25800 & 24900 & 1.53 & 1.49 & 30.6 & 29.7 & 10.2 & 9.9 \\
\hline Boxer (f) & 25.0 & 1.17 & 18.26 & 0.86 & 0.83 & 172.0 & 166.0 & 32400 & 30900 & 1.29 & 1.25 & 25.8 & 24.9 & 8.6 & 8.3 \\
\hline Brindle Boxer & 35.0 & 1.26 & 22.05 & 1.08 & 1.03 & 216.0 & 206.0 & 29400 & 27000 & 1.62 & 1.55 & 32.4 & 30.9 & 10.8 & 10.3 \\
\hline Tawny Boxer & 30.0 & 1.26 & 23.92 & 0.98 & 0.90 & 196.0 & 180.0 & 29400 & 27000 & 1.47 & 1.35 & 29.4 & 27.0 & 9.8 & 9.0 \\
\hline Doberman Pinscher (m) & 40.0 & 1.42 & 19.84 & 1.18 & 1.18 & 236.0 & 236.0 & 35400 & 35400 & 1.77 & 1.77 & 35.4 & 35.4 & 11.8 & 11.8 \\
\hline Doberman Pinscher (f) & 30.0 & 1.32 & 17.22 & 0.98 & 0.98 & 196.0 & 196.0 & 29400 & 29400 & 1.47 & 1.47 & 29.4 & 29.4 & 9.8 & 9.8 \\
\hline Giant Schnauzer & 35.0 & 1.40 & 17.86 & 1.08 & 1.09 & 216.0 & 218.0 & 32400 & 32700 & 1.62 & 1.64 & 32.4 & 32.7 & 10.8 & 10.9 \\
\hline Leonberger & 40.0 & 1.60 & 15.63 & 1.18 & 1.26 & 236.0 & 252.0 & 35400 & 37800 & 1.77 & 1.89 & 35.4 & 37.8 & 11.8 & 12.6 \\
\hline $\begin{array}{l}\text { Long-Haired St. Bernard } \\
\qquad(\mathrm{m})^{* \star *}\end{array}$ & 55.0 & 1.40 & 28.06 & 1.46 & 1.39 & 292.0 & 278.0 & 43800 & 41700 & 2.19 & 2.09 & 43.8 & 41.7 & 14.6 & 13.9 \\
\hline $\begin{array}{l}\text { Long-Haired St. Bernard } \\
\text { (f) }{ }^{* * *}\end{array}$ & 50.0 & 1.30 & 59 & 1.37 & 1.27 & 274.0 & 254.0 & 41100 & 38100 & 2.06 & 1.91 & 41.1 & 38.1 & 13.7 & 12.7 \\
\hline Great Pyrenees (m) & 57.0 & 1.62 & 21.72 & 1.50 & 1.53 & 300.0 & 306.0 & 45000 & 45900 & 2.25 & 2.30 & 45.0 & 45.9 & 15.0 & 12.7 \\
\hline Great Pyrenees (f) & 41.0 & 1.44 & 19.77 & 1.20 & 1.21 & 240.0 & 242.0 & 36000 & 36300 & 1.80 & 1.82 & 36.0 & 36.3 & 12.0 & 12.1 \\
\hline New found land $(m)^{* *}$ & 68.0 & 1.40 & 34.69 & 1.68 & 1.55 & 336.0 & 310.0 & 50400 & 46500 & 2.52 & 2.33 & 50.4 & 46.5 & 16.8 & 15.5 \\
\hline New found land $(\mathrm{f})$ ** & 54.0 & 1.30 & 31.95 & 1.44 & 1.32 & 288.0 & 264.0 & 43200 & 39600 & 2.16 & 1.98 & 43.2 & 39.6 & 14.4 & 13.2 \\
\hline Land Seer ${ }^{* * *}$ & 70.0 & 1.60 & 27.34 & 1.72 & 1.69 & 344.0 & 338.0 & 51600 & 50700 & 2.58 & 2.54 & 51.6 & 50.7 & 17.2 & 16.9 \\
\hline Alaskan Malmute $(\mathrm{m})$ & 38.0 & 1.28 & 23.19 & 1.14 & 1.09 & 228.0 & 218.0 & 34200 & 32700 & 1.71 & 1.64 & 34.2 & 32.7 & 11.4 & 10.9 \\
\hline Alaskan Malmute $(f) * * *$ & 34.0 & 1.16 & 25.27 & 1.06 & 0.97 & 212.0 & 194.0 & 31800 & 29100 & 1.59 & 1.46 & 31.8 & 29.1 & 10.6 & 9.7 \\
\hline Eskimo Dog $(m)^{* *}$ & 50.0 & 0.90 & 61.72 & 1.37 & 1.04 & 274.0 & 208.0 & 41100 & 31200 & 2.06 & 1.56 & 41.1 & 31.2 & 13.7 & 10.4 \\
\hline Eskimo Dog $(\mathrm{f})^{* *}$ & 48.0 & 0.80 & 75.00 & 1.33 & 0.96 & 266.0 & 192.0 & 39900 & 28800 & 2.00 & 1.44 & 39.9 & 28.8 & 13.3 & 9.6 \\
\hline Greenland & 30.0 & 1.10 & 24.79 & 0.98 & 0.89 & 196.0 & 178.0 & 29400 & 26700 & 1.47 & 1.34 & 29.4 & 26.7 & 9.8 & 8.9 \\
\hline Samoyed (m) & 30.0 & 1.20 & 20.83 & 0.98 & 0.93 & 196.0 & 186.0 & 29400 & 27900 & 1.47 & 1.40 & 29.4 & 27.9 & 9.8 & 9.3 \\
\hline Samoyed (f) & 23.0 & 1.06 & 20.47 & 0.82 & 0.76 & 164.0 & 152.0 & 24600 & 22800 & 1.23 & 1.14 & 24.6 & 22.8 & 8.2 & 7.6 \\
\hline Siberian Husky $(\mathrm{m})$ & 27.0 & 1.20 & 18.75 & 0.91 & 0.88 & 182.0 & 176.0 & 27300 & 26400 & 1.37 & 1.32 & 27.3 & 26.4 & 9.1 & 8.8 \\
\hline Siberian Husky (f) & 22.5 & 1.12 & 17.94 & 0.80 & 0.77 & 160.0 & 154.0 & 2400 & 23100 & 1.20 & 1.16 & 24.0 & 23.1 & 8.0 & 7.7 \\
\hline Canaan Dog & 25.0 & 1.20 & 17.36 & 0.86 & 0.84 & 172.0 & 168.0 & 25800 & 25200 & 1.29 & 1.26 & 25.8 & 25.2 & 8.6 & 8.4 \\
\hline Portuguese Water Dog $(\mathrm{m})$ & 25.0 & 1.14 & 19.24 & 0.86 & 0.82 & 172.0 & 164.0 & 25800 & 24600 & 1.29 & 1.23 & 25.8 & 24.6 & 8.6 & 8.2 \\
\hline Portuguese Water Dog (f) & 22.0 & 1.04 & 20.34 & 0.79 & 0.73 & 158.0 & 146.0 & 23700 & 21900 & 1.19 & 1.14 & 23.7 & 21.9 & 7.9 & 7.3 \\
\hline Fila Brasileiro*** & 50.0 & 1.30 & 29.59 & 1.37 & 1.27 & 274.0 & 254.0 & 41100 & 38100 & 2.06 & 1.91 & 41.1 & 38.1 & 13.7 & 12.7 \\
\hline Pyrenees Mastiff*** & 70.0 & 1.60 & 27.34 & 1.72 & 1.69 & 344.0 & 338.0 & 51600 & 50700 & 2.58 & 2.54 & 51.6 & 50.7 & 17.2 & 16.9 \\
\hline Spanish Mastiff** & 60.0 & 1.40 & 30.61 & 1.55 & 1.45 & 310.0 & 290.0 & 46500 & 43500 & 2.33 & 2.18 & 46.5 & 43.5 & 15.5 & 14.5 \\
\hline $\begin{array}{l}\text { Austrian Short-Haired } \\
\text { Pinscher }\end{array}$ & 18.0 & 1.00 & 18.00 & 0.69 & 0.64 & 138.0 & 128.0 & 20700 & 19200 & 1.04 & 0.96 & 20.7 & 19.2 & 6.9 & 6.4 \\
\hline Kyiishii $(\mathrm{m})^{* * *}$ & 30.0 & 1.08 & 25.72 & 0.98 & 0.88 & 196.0 & 176.0 & 29400 & 26400 & 1.47 & 1.32 & 29.4 & 26.4 & 9.8 & 8.8 \\
\hline Kyiishii (f) ** & 30.0 & 0.96 & 32.55 & 0.98 & 0.83 & 196.0 & 166.0 & 29400 & 24900 & 1.47 & 1.25 & 29.4 & 24.9 & 9.8 & 8.3 \\
\hline Norbottens pets & 15.0 & 0.80 & 23.43 & 0.61 & 0.52 & 122.0 & 104.0 & 18300 & 15600 & 0.92 & 0.78 & 18.3 & 15.6 & 6.1 & 5.2 \\
\hline Hokkaidoken & 25.0 & 1.00 & 25.00 & 0.86 & 0.77 & 172.0 & 154.0 & 25800 & 23100 & 1.29 & 1.16 & 25.8 & 23.1 & 8.6 & 7.7 \\
\hline Tosa*** & 37.5 & 1.20 & 26.04 & 1.13 & 1.04 & 226.0 & 208.0 & 3310 & 31200 & 1.70 & 1.56 & 33.9 & 31.2 & 11.3 & 10.4 \\
\hline Akita Inu (m) & 40.0 & 1.42 & 19.84 & 1.18 & 1.18 & 236 & 236 & 35400 & 35400 & 1.77 & 1.77 & 35.4 & 35.4 & 11.8 & 11.8 \\
\hline Akita Inu (f) & 35.0 & 1.32 & 20.09 & 1.08 & 1.06 & 216.0 & 212.0 & 32400 & 31800 & 1.62 & 1.59 & 32.4 & 31.8 & 10.8 & 10.6 \\
\hline Sanshu (m) & 25.0 & 1.10 & 20.66 & 0.86 & 0.81 & 172.0 & 162.0 & 25800 & 24300 & 1.29 & 1.23 & 25.8 & 24.3 & 8.6 & 8.1 \\
\hline Sanshu (f) & 20.0 & 1.00 & 20.00 & 0.74 & 0.68 & 148.0 & 136.0 & 22200 & 20400 & 1.11 & 1.02 & 22.2 & 20.4 & 7.4 & 6.8 \\
\hline Aidi (m) & 30.0 & 1.24 & 19.51 & 0.98 & 0.94 & 196.0 & 188.0 & 29400 & 28200 & 1.47 & 1.41 & 29.4 & 28.2 & 9.8 & 9.4 \\
\hline Aidi (f) & 30.0 & 1.10 & 24.79 & 0.98 & 0.89 & 196.0 & 178.0 & 29400 & 26700 & 1.47 & 1.34 & 29.4 & 26.7 & 9.8 & 8.9 \\
\hline
\end{tabular}




\begin{tabular}{|c|c|c|c|c|c|c|c|c|c|c|c|c|c|c|c|}
\hline Cao Serra Da Estrela*** & 50.0 & 1.44 & 24.11 & 1.32 & 1.34 & 274.0 & 268.0 & 41100 & 40200 & 2.06 & 2.01 & 41.1 & 40.2 & 13.7 & 13.4 \\
\hline $\begin{array}{l}\text { Cao De Castro Laboreiro } \\
(\mathrm{m})^{* * *}\end{array}$ & 40.0 & 1.20 & 27.78 & 1.18 & 1.08 & 236.0 & 216.0 & 35400 & 32400 & 1.77 & 1.62 & 35.4 & 32.4 & 11.8 & 10.8 \\
\hline Cao De Castro Laboreiro (f) & 30.0 & 1.14 & 23.08 & 0.98 & 0.90 & 196.0 & 180.0 & 29400 & 27000 & 1.47 & 1.35 & 29.4 & 27.0 & 9.8 & 9.0 \\
\hline Tibetan Mastiff** & 100.0 & 1.60 & 39.06 & 2.18 & 2.04 & 436.0 & 408.0 & 65400 & 61200 & 3.27 & 3.06 & 65.4 & 61.2 & 21.8 & 20.4 \\
\hline Keeshond $(\mathrm{m})^{* *}$ & 30.0 & 0.96 & 32.55 & 0.98 & 0.83 & 196.0 & 166.0 & 29400 & 24900 & 1.47 & 1.25 & 29.4 & 24.9 & 9.8 & 8.3 \\
\hline Keeshond $(f)$ ** & 25.0 & 0.90 & 30.86 & 0.86 & 0.72 & 172.0 & 144.0 & 25800 & 21600 & 1.29 & 1.08 & 25.8 & 21.6 & 8.6 & 7.2 \\
\hline
\end{tabular}

Table 8: Body weight, height, body mass index, body surface areas and therapeutic doses of anticancer drugs in working dogs.

proposed to the various scalings [24]. The fact that all the human BSA formulas applied in this study yield BSA of larger dogs less than that of Cowgill and Drabkin's formula, indicates that human BSA formulas may be used in cancer chemotherapy of dogs. Since human BSA formulas have body height and after having multiplied body height of the dogs by 2, the formulas yielded BSAs of dogs less than that of Cowgill and Drabkin's formula [8], suggesting that the current formula used to estimate BSA of dogs may be inaccurate, because the body height is lacking. Although body surface area and weight were found to be significantly correlated with severity of toxicity [2], relative to large dogs, the prevalence of melphalan, cisplatin and carboplatin toxicosis is greater in small dogs which are at greater risk of developing bone marrow toxicity from melphalan than large dogs, if Cowgill and Drabkin's formula is used [25]. The derived formula $\left(\mathrm{BSA}=\mathrm{BW}^{0.528} \times\right.$ $\mathrm{H}^{0.528} \times \mathrm{K}$ ) where $\mathrm{k}$ is 0.14 yielded BSAs and doses of anticancer agents less than that of all human formulas except Boyd formula. The body weight and height exponents of 0.25-0.30 and 0.425-0.514 of human BSAs used in this study agrees with the report, indicating that indices of body measures are numerous for both humans and animals [26]. Dosing per unit of weight alone is responsible for a large dose of anticancer drug that can cause toxicity. Therefore, the principles of $\mathrm{BW}^{1 / 2}$ to $\mathrm{BW}^{3 / 4}$ across species may be adopted. But $\mathrm{BW}^{3 / 4}$ has been adopted for conversion of data between species [27].

The fact that fourteen out of 18 greyhounds recorded higher BSA values using the new formula shows that dogs with high body weight and high body height yield BSA value higher than that yielded by Cowgill and Drabkin's formula. But Wolfhound BSA $\left(1.18 \mathrm{~m}^{2}\right)$ using the new and Cowgill and Drabkin's formula shows that at the weight (40 kg) and height $(1.42 \mathrm{~m})$, the exponents of the formulas may be approximately same, and at weight higher or lower than $40 \mathrm{~kg}$, but at height lower or higher than $1.42 \mathrm{~m}$ there may be either decrease or increase in BSA value. Since prediction errors are commonly observed in the practical application of allometric scaling, various modifications have been proposed [28]. Therefore, application of Cowgill and Drabkin's formula for calculation of BSA for greyhounds may be preferred to the new formula. But on contrary, toys recorded lower BSA values using the new formula. This may be due to their low body weight relative to low height. Since Affenspincher recorded same value of BSA $\left(0.24 \mathrm{~m}^{2}\right)$ using the new and Cowgill and Drabkin's formula, it implies that at weight less or higher than $3.6 \mathrm{~kg}$ and height less or higher than $0.76 \mathrm{~m}$, BSA value may either be increased or decreased in this breed of dog. Therefore, application of the new formula may be preferred for this dog. However, obtaining of lower doses of vincristine $\left(0.6 \mathrm{mg} / \mathrm{m}^{2}\right)$ and mechlorethamine $\left(0.56 \mathrm{mg} / \mathrm{m}^{2}\right)$ yielded by Cowgill and Drabkin and the new formula disagree with the reported therapeutic doses of vincristine $\left(0.75 / \mathrm{m}^{2}\right)$ and mechlorethamine $\left(3 \mathrm{mg} / \mathrm{m}^{2}\right)$ [7]. When doxorubicin doses were calculated using BSA rather than body weight, drug concentrations and toxicoses were greater in smaller dogs [1]. The similar BSA $\left(0.24 \mathrm{~m}^{2}\right)$ yielded by the new and Cowgill and Drabkin's formula for Affenpinscher with body weight $(3.6 \mathrm{~kg})$ and height $0.38 \mathrm{~m}$ as well as BSA $\left(0.74 \mathrm{~m}^{2}\right)$ of Airedale with body weight
$(20.0 \mathrm{~kg})$ and height, $(1.18 \mathrm{~m})$ show that toys and terriers may share some common pharmacokinetic parameters that are related to their body functions. The reported therapeutic doses of cyclophosphamide $\left(50 \mathrm{mg} / \mathrm{m}^{2}\right)$ in transmissible venereal tumor (TVT) of dogs [29] carboplatin $\left(300 \mathrm{mg} / \mathrm{m}^{2}\right)$ for osteosarcoma [30], dacarbazine and (200 $\mathrm{mg} / \mathrm{m}^{2}$ ) for canine hemangiosarcoma [31] corroborate the present calculated therapeutic values for cyclophosphamide, carboplatin and dacarbazine, respectively. The similarity in the estimated values may be accounted for by similarity in the exponents of their body weight and height. But the exponent (0.67) has a highly significant correlation to body weight [27]. Different values of BSAs and anticancer doses maybe, in the present analysis due to variation in exponents (0.67) and (0.528) reported by Cowgill and Drabkin and this paper, respectively. Therefore, it is reasonable to use a formula in which body weight and height are not ignored, to more accurately normalize chemotherapeutic exposure and reduce or avoid toxicosis in dogs. The relevance of the new formula derived from human BSA formulas which yielded results lower than Cowgill and Drabkin's formula cannot be over emphasized. Although anticancer overdosing may be easily recognized, underdosing may occur in $30 \%$ of cancer patients [32]. This is because the predicted values for humans are heavily depended on certain species for example, dog [24], which has similar stomach morphology and emptying characteristics with human [33]. Higher BMI $>25 \mathrm{~kg} / \mathrm{m}^{2}$ recorded for Irish Wolf Hound, Chinese Imperial Ch'ln (giant), Chinese Imperial Ch'ln (classic), Chinese Imperial Ch'ln (miniature), Chinese Imperial Ch'ln (Sleeve), Poodle (standard), Great Spitz, French Bulldog, Poodle (miniature) and Chinese Temple dog (miniature) may portend higher tendency for obesity, hypertension, coronary artery disease, diabetes and cancer in these breeds. Although improved nutrition may cause an increase in body size in carnivores, while increased ambient temperature may result in decreased body size as expected by Bergamann's rule, majority of hunting and working dogs yield lower BSA values using the new formula. This shows that high body weight with corresponding body height yield lower BSA value. Also, majority of hunting and working dogs are tall and weigh relatively heavier than greyhounds hence, 23 and 25 hunting and working dogs, respectively recorded BMI greater than $30 \mathrm{~kg} / \mathrm{m}^{2}$. Breed differences in susceptibility to diabetes mellitus in dogs is genetic as observed in the UK dog population. The affected breeds are Samoyed, Tibetan Terrier, Cairn Terrier, Miniature Schnauzer, Yorkshire Terrier, Border Terrier, Labrador Retriever, Golden Retriever, German Shepherd dog and Boxer [34], Bichon Fries, Border Collie, Border Terrier, Collie, Dachshound, English Setter, Poodle, Schnauzer, English Springer Spaniard, Staffordshire Bull Terrier, Weimaraner, Welsh Springer Spaniel, Cavalier King Charles Spaniel, Cocker Spaniel, Doberman, Jack Russell Terrier, Rottweiler and West Highland Terrier [28]. Overweight prevalence of $30 \%$ or more was found for Beagle, Shetland Sheepdog and Dalmatian. BMI $>25 \mathrm{~kg} / \mathrm{m}^{2}$ suggest overweight and $\mathrm{BMI}>30 \mathrm{~kg} / \mathrm{m}^{2}$ is indicative of obesity in humans. However, dog is said to be overweight when body weight is greater than $15 \%$ of their optimal body weight and obese when body weight exceeds $30 \%$ of optimal [35]. The special attributes of the dog for comparative medicine are large 
Citation: Saganuwan SA (2017) Derivation of a Unique Body Surface Area (Bsa) Formula for Calculation of Relatively Safe Doses of Dog and Human Anticancer Drugs. J Cancer Sci Ther 9: 690-704. doi:10.4172/1948-5956.1000493

body size, remarkable genotype homogeneity compared with human ethnic group, controllable breeding and nutrition and applicability of clinical techniques between children and dogs [30]. The similar BSAs for Kardian Bear $\operatorname{dog}\left(0.79 \mathrm{~m}^{2}\right)$ and Brigue Ariergeois $\left(0.79 \mathrm{~m}^{2}\right)$ as well as Braque D'Aurvergne female $\left(0.74 \mathrm{~m}^{2}\right)$ and French Spaniel female 0.74 $\mathrm{m}^{2}$ ) show that doses of anticancer drugs are calculated as a function of several pharmacokinetic parameters, including volume of distribution $(\mathrm{Vd})$ and total body clearance $(\mathrm{Cl})$. However, the variation in body size over an order of magnitude as shown in grey hounds, toys, companions, terriers, hunting and working dogs, may be more accurately described as a non-proportional function of body weight [26]. Since metabolic rate, body surface area (BSA), organ blood flow and glomerular filtration rate (GFR) depend on body weight [27], pharmacokinetic parameters and appropriate drug doses scale allometrically with body weight [36,37]. Allometric equation of the general form is: $y=a W^{2}$, where $\mathrm{y}$ is the scalable variable, a is the mass coefficient, $\mathrm{W}$ represents body weight, and $b$ is the mass exponent. The relationship between pharmacokinetic terms and body size is related to a single mass exponent [38]. When a pharmacokinetic parameter is available from one species, the allometric equation: $Y_{\text {human }}=Y_{\text {animal }}\left(\frac{W_{\text {human }}}{Y_{\text {animal }}}\right)^{b}$ can be used to predict the value of the parameter in humans. The value of $b$ can be related to mean residence time (MRT $=0.25), V d=1.0$, blood flow, renal and hepatic clearance (0.75). For multiple species $\log \mathrm{Y}=\log (\mathrm{a})+\mathrm{b} \log (\mathrm{W})$, whereas $\mathrm{b}$ derived from compound specific data can be used. The similarities between dog and human cancers are related to cancer-association genes such as MET, IGFIR, mTor, KIT [39] and BCR-Abl translocation in myelogeneous leukemia and RBI in human [40]. Therefore, comparative therapeutics will eliminate highly toxic and non-effective anticancer drugs [41]. Metabolic rate being related with BSA depends on body weight and exponent (1/4) [42] which further predicts pharmacokinetic mass exponents for clearance (3/4), elimination half-life $(\mathrm{t} 1 / 2,1 / 4)$, and volume of distribution. Clearance with BSA also scale with the $2 / 3$ power. Intraspecific studies in mammals have correlated the allometry of physiological parameters with that of drug disposition [43] suggesting that obesity could increase BSA value [44].

\section{Conclusion}

Boyd formula can be used for estimating body surface area of dogs, since it provides low doses of anticancer agents, whereas this paper, Saganuwan and Ndakotsu, Fujimoto and Mosteller formulas provide relatively averaged doses for larger breeds of dogs. DuBois, Takashira and Wang formulas can serve as alternatives. The rest of human body surface area formulas provide BSAs lower than the original body surface area formulas of dog. Human BSA formulas provide moderate doses of anticancer agents that may be effective and less toxic. Toys, companions and terriers have lower BSAs in comparison with greyhounds using Saganuwan's formula. Whereas majority of greyhounds recorded higher BSA values using Saganuwans's formula. The difference in BSA values might depend on the weight, height and exponents of the BSA formulas used. Hunting and working dogs have lower BSA values using Saganuwan's formula. Some breeds from the two categories have high risk of becoming obese, hypertensive and diabetic. But Irish Wolf hound, Affenspincher, Airedale terrier, Irish setter, Briquette, French Spaniel, Braque D’ Aurvergne (female), Brique Ariegeois, Weimaraner (male), Drahthaar (female), Red-tick Coon Hound, Bluetick Coon Hound (male), Rodhesia Ridgeback (female), Smooth-Haired Istrian Hound, Billy (male), Kuvasz, Maremma Sheep dog, Rafeiro Do Alentejo, Austrian Shepherd, Doberman pinscher (male and female) and Akita Inu (female) recorded same values of
BSAs and doses of anticancer drugs using Cowgill and Drabkin's as well as the new derived formula.

\section{Acknowledgement}

I sincerely thank Kehinde Ola Emmanuel of National Open University, Nigeria for typing this work.

\section{Availability of Data Materials}

All the data supporting our findings are contained within the manuscript.

\section{Conflict of Interest}

The author declares that he has no conflict of interest.

\section{Author's Contributions}

The author SAS designed the study, generated, analysed and interpreted the data, drafted, revised and approved the version of the manuscript to be published.

\section{Ethical Approval}

This article does not contain any study with human or animal, but rather establishes body surface areas and doses of some anticancer drugs for various breeds of dogs.

\section{Funding}

This study was self funded.

\section{References}

1. Arrington KA, Legendre AM, Tabeling GS, Frazier DL (1998) Comparison of body surface area-based and weight-based dosage protocol for doxorubicin administration in dogs. Am J Vet Res 55: 1587-1592.

2. Belo VS, Werneck GL, Da Silva ES, Barbosa DS, Struchiner CJ, et al. (2015) Population estimation methods for free ranging dogs: A systematic review. Plos One 10: e0144830.

3. Miller AA (2002) Body surface area in dosing anticancer agent: Scratch the surface. J Natur Cancer Instit 94: 1822-1823.

4. Saganuwan SA (2012) Principles of pharmacological calculations, (1st edn) Ahmadu Bello University Press, Zaria, Nigeria. p. 529.

5. DuBois D, DuBois EF (1916) A formula to estimate the approximate surface area of height and weight be known. Arch Intern Med 17: 863-871.

6. Wang Y, Moss J, Thisted R (1992) Predicators of body surface area. J Clin Anesthesiol 4: 4-10.

7. Back AR, Schleis SE, Smrkovski OA, Lee I, Smith AN, et al. (2015) Mechlorethamine, vincristine, melphalan and prednisone (MOMP) for the treatment of relapsed lymphoma in dogs. Vet Compar Oncol 13: 398-408.

8. Cowgill GR, Drabkin D (1927) Determination of a formula for the surface area of the dog together with a consideration of formulae available for other species. Am J Physiol 81: 36-61.

9. Gold LS, Slone TH, Manley NB (2002) Misconceptions about the causes of cancer. Risk Controversy Series. The Eraser Institute, Centre for Studies in Risk, Regulation and Environment, Vancouver, British Colombia, Canada 3: $1-141$.

10. Saganuwan SA, Ndakotsu AM (2015) Standardization and soring of the body surface area (BSA) formulas for calculation of the doses of anticancer agents for cancer patients from the North-Western Nigeria. J Cancer Sci Ther 7: 02-018.

11. Mosteller RD (1987) Simplified calculation of body surface area. N Eng J Med 317: 1098.

12. Haycok GB, Schwartz GJ, Wisotsky DH (1978) Geometric method for measuring body surface area: $A$ height weight formula validated in infants, children and adults. J Paediatric 93: 62-66.

13. Gehan EA, George SL (1970) Estimation of human body surface area from height and weight. Cancer Chemother Reprod 54: 225-235.

14. Boyd E (1935) The growth of the surface area of the human. University of Minesota, press, USA.

15. Freireich EJ, Gehan EA, Rall DP (1996) Quantitative comparison of toxicity of anticancer agents in mouse, rat, hamster, dog, monkey and man. Cancer Chemother Report 50: 219-223. 
Citation: Saganuwan SA (2017) Derivation of a Unique Body Surface Area (Bsa) Formula for Calculation of Relatively Safe Doses of Dog and Human Anticancer Drugs. J Cancer Sci Ther 9: 690-704. doi:10.4172/1948-5956.1000493

16. Fujimoto S, Watanabe T, Sakamato A (1968) Studies on the physical surface area of Japanese. Is. calculation formulae in three stages over all ages. Nippon Eiseigoku Zassho 5: 443-450.

17. Pugnetti G (1980) Simon and Schuster's Guide to Dogs, Simons and Schuster, New York, USA 313-323.

18. Sandhu HS, Rampal S (2006) Essentials of veterinary, pharmacology and therapeutics, Kalyani Publishers, New Delhi, India. p. 1529.

19. Aliu YO (2001) Veterinary pharmacology (1st edn), Tamaza Publishing Company Ltd., Zaria, Nigeria 814.

20. Khan CM (2010) The Merck Veterinary Manual, (10th edn), Merck \& Co Inc. USA. 2945.

21. Price GS, Frazier DL (1998) Use of body surface area (BSA) based dosages to calculate chemotherapeutic drug dose in dogs: 1. Potential problems with current BSA formulae. J Vet Intern Med 12: 267-271.

22. Sturgess K (2012) Pocket Handbook of Small Animal Medicine, CRC Press, Tailor and Francis, USA 192

23. Howell DC (2004) Fundamental Statistics for the Behavioural Science, (5th edn)., Thomson Wadsworth, Belmonth USA 570.

24. Tang $H$, Mayersohn M (2005) A novel model for prediction of human drug clearance by allometric scaling. Drug Metab Dispos 33: 1297-1303.

25. Page RL, Macy DW, Thrall DE (1988) Unexpected toxicity associated with use of body surface area for dosing Melphalan in dog. Cancer Res 48: 288-290.

26. Mordent $\mathrm{J}$ (1985) Forecasting rephalosporin and monobactam antibiotic halflives in humans from data collected in laboratory animals. Antimicrob Agent Chemother 27: 887-891.

27. Kleiber M (1947) Body size and metabolic rate. Physiol Rev 27: 511-541.

28. Catchpole B, Adams JP, Holder AL (2013) Genetics of cancine diabetes mellitus: Are the diabetes susceptibility genes identified in humans in breed susceptibility to diabetes mellitus in dogs? Vet J 195: 139-147.

29. Al-Vitalaru B, Crangmu D, Polter G (2017) Comparative therapeutic approach of canine transmissible venereal tumor (TVT). Faculty of Veterinary Medicine Bucharest. pp. 1-8.

30. Duffy D, Selmic LE, Kendall, AR, Powers BE (2015) Outcome following treatment of soft tissue and visceral extraskeletal osteosarcoma in 33 dogs: 2008-2013. Vet. Comparat Oncol 15: 46-54
31. Finotello R, Stefanello D, Zini E, Marconato L (2015) comparison of doxorubicincyclophophamide with doxorubicin-dacarbazine for the adjuvant treatment of canine hemangiosarcoma. Vet Comparat Oncol 15: 25-35.

32. Gurney $\mathrm{H}$ (2002) how to calculate the dose of chemotherapy. Br J Cancer 86 : 1297- 1302

33. Kararli TT (1995) Comparison of the gastrointestinal anatomy, physiology, and biochemistry of humans and commonly used laboratory animals. Biopharmaceutic Drug Dispos 16: 351-380.

34. Catchpole B, Kennedy LJ, Davison LJ (2007) Canine diabetes mellitus: From phenotype to genotype. J Small Animal Pract 49: 4-10.

35. Burkholder WJ, Toll PW, MS Thatcher, CD, Reimillard RL, Roudebush (2000) Small animal clinical nutrition, (4th edn), Morris Institute: Topeka KS 401-430.

36. Courcier EA, Thomson RM, Mellor DJ, Yam PS (2010) An epidemiological study of environmental factors associated with canine obesity. J Small Animal Pract 51: $362-367$

37. Mahmood I (1998) Interspecies scaling: Predicting volumes, mean residence time and elimination half-life. Some suggestions. J Pharm Pharmacol 50: 493-499.

38. Mahmood I (2006) Prediction of drug clearance in children from adults: A comparison of several allometric methods. Br J Clin Pharmacol 61: 545-557.

39. Lave T, Coassolo P, Ubeaud G (1996) Interspecies scaling of bosentan, a new endothelin receptor antagonist and integration of in vitro data into allometric scaling. Pharmaceutic Res 13: 97-101.

40. Paoloni M, Khanna C (2008) Translation of new cancer treatments from pet dogs to humans. Natur Rev Cancer 8: 147-156.

41. Kouno T, Katsumata N, Mukai H (2003) Standardization of body surface area (bsa) to calculate the dose of anticancer agents in Japan. Japanese $\mathrm{J}$ Clin Oncol 33: 309-313

42. Anderson BJ, Allegaert K, Holford NHG (2006) Population clinical pharmacology of children: Modelling covariate effects. Eur J Pediatric 165: 819-829.

43. Yom-Tov $Y$ (2003) Body sizes of carnivores commensal with humans have increased over the past 50 years. Functional Ecol 17: 323-327.

44. Lund EM, Armstrong PJ, Kurk CA (2006) Prevalence and risk factors fo obesity in adult dogs from private US veterinary practice. Int J Appl Res Vet Med 4: 177-186 